\title{
Fibroblast subtypes define a metastatic matrisome in breast cancer
}

\author{
Heather M. Brechbuhl, ${ }^{1}$ Alexander S. Barrett, ${ }^{2}$ Etana Kopin, ${ }^{1}$ Jaime C. Hagen, ${ }^{1}$ Amy L. Han, ${ }^{1}$ \\ Austin E. Gillen, ${ }^{3}$ Jessica Finlay-Schultz, ${ }^{4}$ Diana M. Cittelly, ${ }^{4}$ Philip Owens, ${ }^{4,5}$ Kathryn B. Horwitz, ${ }^{4,6}$ \\ Carol A. Sartorius, ${ }^{3}$ Kirk Hansen, ${ }^{2}$ and Peter Kabos ${ }^{1}$ \\ DDivision of Medical Oncology, Department of Medicine, ${ }^{2}$ Department of Biochemistry and Molecular Genetics, ${ }^{3}$ RNA \\ Bioscience Initiative, and ${ }^{4}$ Department of Pathology, University of Colorado Denver Anschutz Medical Campus, Aurora, \\ Colorado USA. ${ }^{5}$ Research Service, Department of Veterans Affairs, Eastern Colorado Health Care System, Aurora, \\ Colorado, USA. 'Division of Endocrinology, Department of Medicine, University of Colorado Denver Anschutz Medical \\ Campus, Aurora, Colorado, USA.
}

Small primary breast cancers can show surprisingly high potential for metastasis. Clinical decisionmaking for tumor aggressiveness, including molecular profiling, relies primarily on analysis of the cancer cells. Here we show that this analysis is insufficient - that the stromal microenvironment of the primary tumor plays a key role in tumor cell dissemination and implantation at distant sites. We previously described 2 cancer-associated fibroblasts (CAFs) that either express (CD146+) or lack (CD146-) CD146 (official symbol MCAM, alias MUC18). We now find that when mixed with human breast cancer cells, each fibroblast subtype determines the fate of cancer cells: CD146- fibroblasts promoted increased metastasis compared with $\mathrm{CD} 146^{+}$fibroblasts. Potentially novel quantitative and qualitative proteomic analyses showed that CD146+ CAFs produced an environment rich in basement membrane proteins, while CD146- CAFs exhibited increases in fibronectin 1, lysyl oxidase, and tenascin C, all overexpressed in aggressive disease. We also show clinically that CD146- CAFs predicted for likelihood of lymph node involvement even in small primary tumors $(<5 \mathrm{~cm})$. Clearly small tumors enriched for CD146- CAFs require aggressive treatments.

Conflict of interest: The authors have declared that no conflict of interest exists.

Copyright: () 2020, American Society for Clinical Investigation.

Submitted: June 3, 2019

Accepted: January 29, 2020

Published: February 6, 2020.

Reference information: /CI Insight. 2020;5(4):e130751.

https://doi.org/10.1172/jici.

insight.130751.

\section{Introduction}

Development of breast cancer metastasis requires a tumor microenvironment (TME) that allows cancer cells to invade, migrate, and ultimately disperse from the primary disease site. Dysregulation of both structural (i.e., collagen I and IV, proteoglycans, and glycoproteins) and matricellular components of the TME (i.e., growth factors, metalloproteinases, tissue inhibitors of metalloproteinases, chemokines, and cytokines) has been linked with development of metastases in multiple cancer types $(1,2)$. For example, in breast tissue, the glycoprotein tenascin C (TNC) is often overexpressed at the invasive front of aggressive and metastatic breast tumors $(3,4)$. TNC uses its fibronectin 1 (FN1) and EGF-like repeat domains to change cancer cell adhesion, proliferation, and migration. TNC is one of many proteins and signaling molecules in the TME that are influenced by the dynamic relationship between cancer cells and stroma, which both contribute to extracellular matrix (ECM) composition. Ultimately, these interactions help shape how aggressive a tumor becomes, and they can initiate and maintain signaling pathways that support invasion and metastasis formation.

Several stromal cell types contribute to the establishment and maintenance of the TME; however, most commonly, cancer-associated fibroblasts (CAFs) represent the predominant nonepithelial component of primary breast cancers. We and others have shown that CAFs represent a heterogenous cell population known to influence treatment response and metastatic potential of cancer cells by secreting matricellular proteins and signaling molecules $(5,6)$. Previously we described 2 subtypes of breast cancer fibroblasts, based on the presence or absence of the melanoma cell adhesion molecule (MCAM; alias, MUC18 or CD146) (5) and demonstrated that CD146- CAFs promoted loss of estrogen receptor (ER) expression and decreased response to antiendocrine therapy in a model of $\mathrm{ER}^{+}$breast cancer. Such mounting evidence lends to the hypothesis that improved understanding of CAF subtypes and their influence on ECM composition will ultimately lead to an improvement in breast cancer treatment strategies. To that end, our lab and others have begun isolating and defining subpopulations of CAFs based on several cell markers, including 
caveolin 1, $\alpha$-smooth muscle actin, fibroblast-specific protein $1, \alpha$ - and $\beta$-platelet-derived growth factor, vimentin, and others $(7,8)$.

Here, we hypothesized that influence from CAF subtypes, identified by the presence or absence of CD146 expression, would drive the production of qualitatively different TME matrices in the presence of the same breast cancer cell types. In order to describe the contributions of each CAF subtype to the TME, we developed a potentially new metastatic model of $\mathrm{ER}^{+}$disease and used an innovative ECM-focused mass spectrometry-based approach (9-12). Here, we describe how CD146- CAFs produced an increased metastasis-enhancing TME compared with the CD146+ CAF-derived TME. We further show that CD146 CAF-dependent cancer cell invasion and metastatic phenotype are dependent on TNC expression and epidermal growth factor receptor (EGFR) pathway activation.

\section{Results}

The TME of tumors bearing CD146- CAFs or CD146 $C A F$ s are defined by unique ECM profiles. We injected a mixture of 150,000 ZS-green-labeled ER ${ }^{+}$MCF-7 cells with either 150,000 CD146+ CAFs or CD146- CAFs into the mammary fat pad of intact female NOD/SCID- $\gamma$ (NSG) mice supplemented with 1 mg estrogen pellets. All tumors were collected at the same time with restriction of any single tumor reaching $2 \mathrm{~cm}$ in size by caliper measurement. This corresponded to approximately 5 weeks for establishment of primary tumors and 6 weeks of measurable growth. To determine the effects of CD146 ${ }^{+}$CAFs and CD146 CAFs on the TME, we modified the standard ECM-focused mass spectrometry-based approach for quantifying ECM proteins. We used a library of stable isotope-labeled reporter peptides containing a mixture of human-specific, mouse-specific, and shared species ECM probes. This unique method afforded us the ability to quantitatively measure and specifically attribute host versus human contributions to the TME. Liquid chromatography-select reaction monitoring (LC-SRM) was applied to quantitatively assess differences in the abundance of ECM and ECM-associated components of the TME. In order to obtain the most comprehensive analysis of these components, each sample was processed using 3 serial extractions, including cellular (CHAPS with high salt), soluble ECM (guanidine hydrochloride), and insoluble ECM $\left(\mathrm{NH}_{2} \mathrm{OH}\right.$ digestion) fractions. Each of the 3 fractions was spiked with stable isotope-labeled peptide standards specific to ECM and ECM-associated proteins at a known concentration (Figure 1A and ref. 11). This experiment resulted in the absolute quantification of $127 \mathrm{ECM}$ and ECM-associated proteins of interest in our tumor samples: 24 specific to human, 36 specific to mouse, and 67 with shared peptide sequences (Figure $1 \mathrm{~B}$ ). CD146 $\mathrm{CAF}$ - and CD146- $\mathrm{CAF}$-derived tumors were distinguished from one another based on hierarchical clustering of the complete proteome - all 127 proteins (Supplemental Figure 1; supplemental material available online with this article; https://doi.org/10.1172/jci.insight.130751DS1) - as well as clustering based on the human or shared proteins. Interestingly, the CAF-derived tumors were not hierarchically clustered based on the mouse proteome. Principal component analysis (PCA) showed the establishment of 2 unique TME profiles dependent on CAF subtype (Figure 1C). These data suggest that in our model the human (i.e., CAFs and tumor cells) secretome dictated the TME composition to a greater extent than the host (i.e., mouse)

The TME of CD146-CAF-derived tumors is enriched for prometastatic proteins. We used functional matrisome analysis to group ECM proteins according to their Gene Ontology functional classification as previously described $(10,12)$. Organizing the data in this way allowed us to observe broad differences in $\mathrm{CD}_{146} \mathrm{CAF}^{-}$ versus $\mathrm{CD} 146^{+} \mathrm{CAF}$-derived TMEs. In $\mathrm{CD} 146^{-} \mathrm{CAF}$-influenced tumors, the analysis revealed significantly decreased abundance of basement membrane proteins and significantly increased abundance of ECM regulators (e.g., lysyl oxidase, LOX) and structural ECM proteins (e.g., FN1, TNC, collagens) (Figure 2A). These changes indicate that $\mathrm{CD} 146^{-} \mathrm{CAF}$-containing tumors underwent substantial ECM remodeling supportive of cancer cell invasion, disease progression, and metastatic development. Of the 127 detected proteins, 73 proteins were identified as having greater than 1.5 -fold difference $(P<0.055)$ between $\mathrm{CD} 146^{+} \mathrm{CAF}$ and CD146- CAF tumors (Figure 2B and Supplemental Table 1). In MCF-7/CD146 ${ }^{+}$CAF tumors, 48 proteins were expressed significantly more compared with MCF-7/CD146- CAF tumors, 3 human-specific ECM proteins were increased (thrombospondin 1, 2.17-fold, $P=0.009$; COL18A1, 1.96-fold, $P=0.014$; and agrin, expressed only in tumors with $\left.\mathrm{CD} 146^{+} \mathrm{CAFs}\right), 27$ were identified as shared, and 18 were identified as coming from mouse. In contrast, MCF-7/CD146- CAF tumors showed significant increase in 25 proteins, of which 15 were human specific, 10 were identified as shared, and none were from mouse. These data support the conclusion that interactions between the human CD146- CAFs and MCF-7 cells promoted an increasingly reactive TME compared with the interaction between CD146 ${ }^{+}$CAFs and MCF-7 cells. 
A

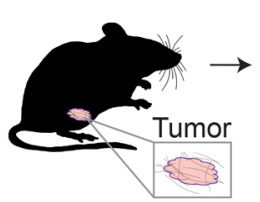

PDX in

CD146(+/-) model
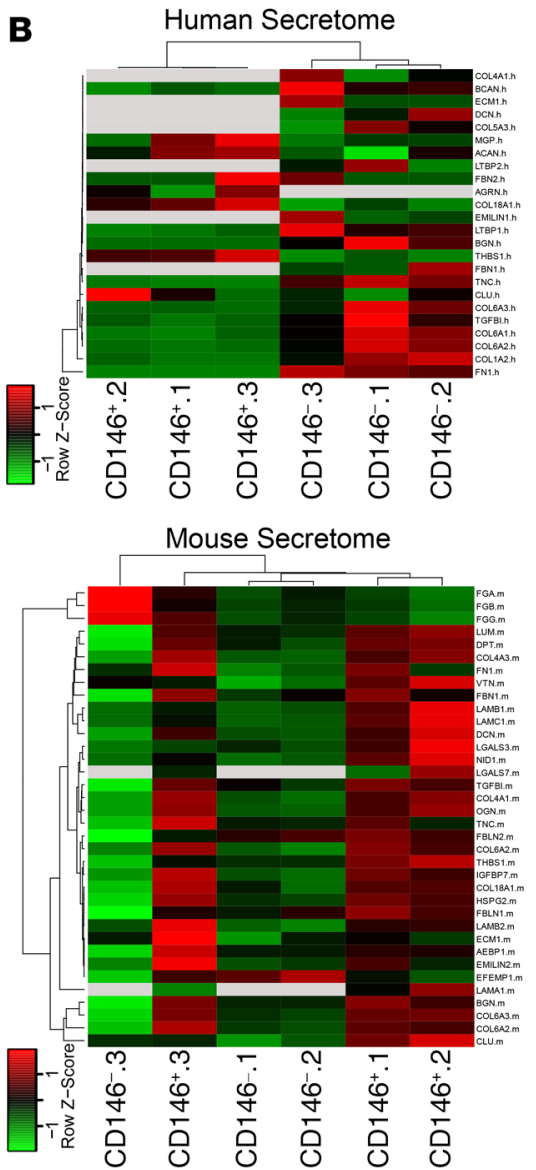

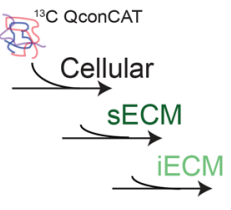

Sequential tissue extraction and fractionation on pellet

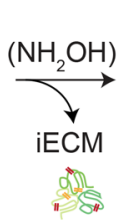
Tryptic digestion
${ }^{13} \mathrm{C}$ QconCATs
with species resolved

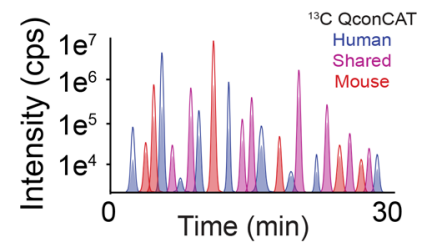

LC-SRM data collection and quantification

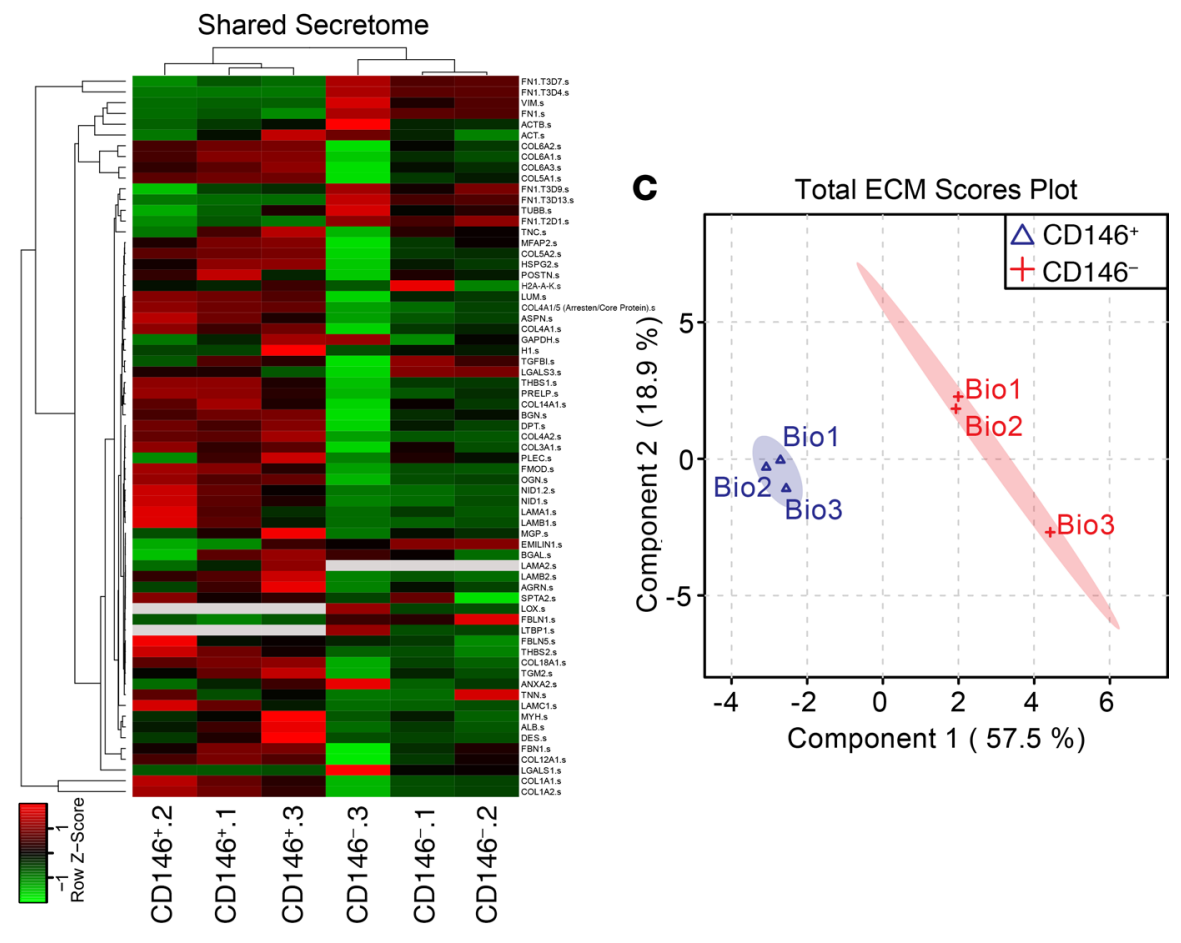

Figure 1. Tumors bearing CD146- or CD146+ CAFs are identified based on their TME proteomic profiles. (A) Pictorial representation of the proteomics approach used to quantitatively measure human-produced versus host-derived proteins in the TME. sECM, soluble extracellular matrix; iECM, insoluble extracellular matrix; QconCAT, quantitative concatemers. (B) Heatmap illustrations show how tumors cluster according to CAF subtype by the human secretome and shared secretome (single asterisk, arrestin/core protein double asterisk, H2A-A-A-K), but not by the mouse secretome. Proteomics was completed in triplicate (CD146+1-.3 and CD146-1-.3). Low to high expression, green to red; gray, no expression. (C) Principal component analysis of total extracellular matrix scores demonstrates distinct TMEs that classify tumors based on CAF subtypes.

MCF-7/CD146 CAF tumors expressed significantly more laminin proteins, which are major components of the basement membrane and act as an important barrier to tumor cell invasion. Laminin $\alpha 1^{\mathrm{M}}$ and laminin $\alpha 2^{\mathrm{S}}$ were restricted to CD146 ${ }^{+} \mathrm{CAF}$ tumors, and laminin $\beta 2^{\mathrm{M}}$, laminin $\gamma 1^{\mathrm{M}}$, laminin $\beta 1^{\mathrm{s}}$, laminin $\beta 2^{\mathrm{S}}$, and laminin $\gamma 1^{\mathrm{S}}$ increased, respectively, by 3.2-fold $(P=0.033), 12.5$-fold $(P=0.049), 12.5$ fold $(P=0.055), 3.6$-fold $(P=0.011)$, and 9.1-fold $(P=0.046)$. In contrast, CD146- CAF-containing tumors expressed significantly more human-specific collagens associated with tumor progression and metastasis. COL4A1 and COL5A3 were detected only in CD146- CAF tumors, and other collagens were strongly upregulated, including COL1A2, 14.8-fold $(P=0.031)$; COL6A1, 10.1-fold $(P=0.021)$; COL6A2, 12.2-fold $(P=0.021)$; and COL6A3, 4.3-fold $(P=0.060)(13-16)$. Furthermore, LOX, which catalyzes formation of collagen cross-links, was expressed exclusively in tumors mixed with CD146 CAFs. Human-produced ECM glycoproteins FN1 and TNC were increased 50-fold and 21-fold, respectively $(P=0.001 ; P=0.002)$ (Figure $2 \mathrm{~B}$ and Supplemental Table 1). Overexpression of FN1 has been 

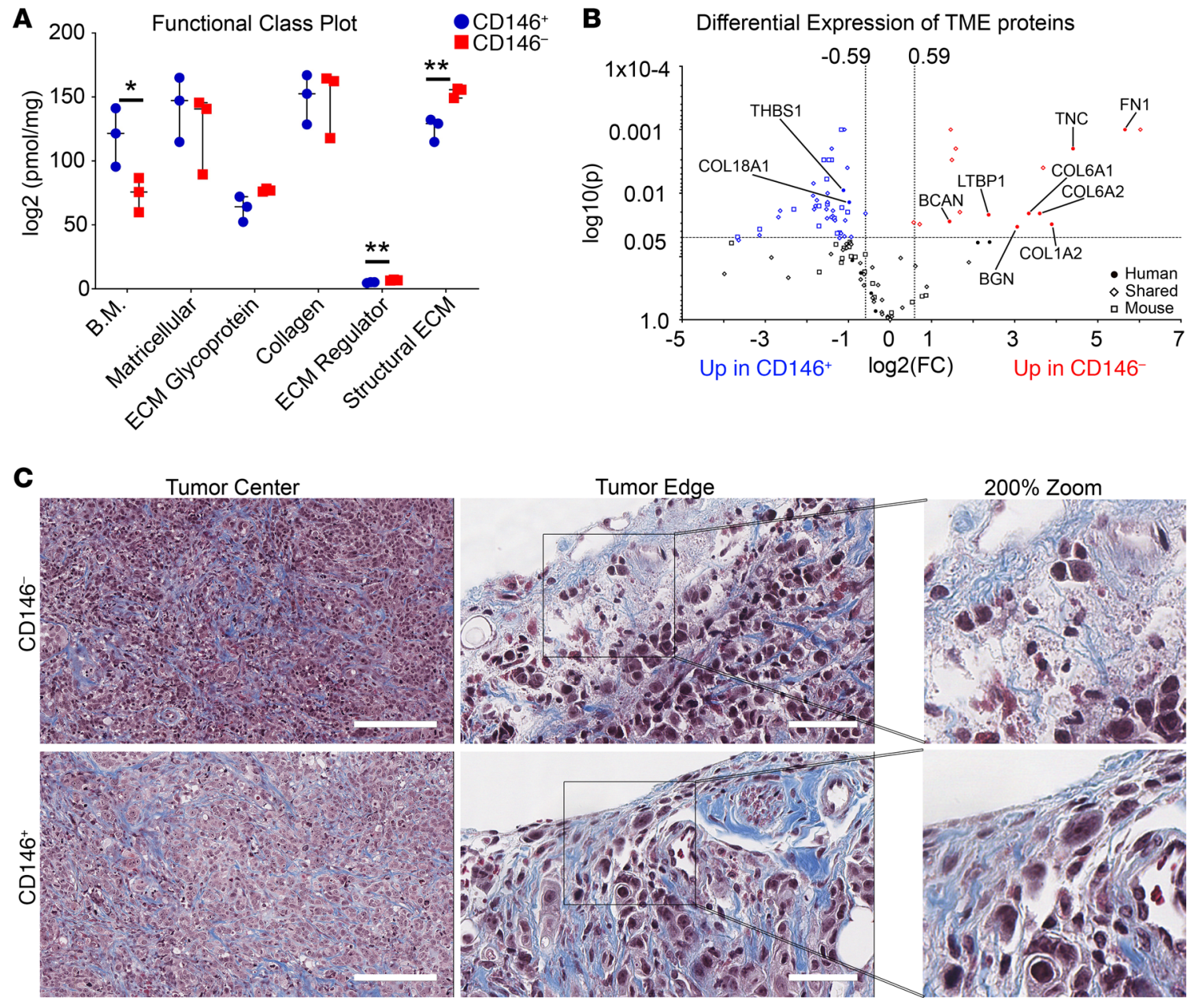

Figure 2. Tumors bearing CD146- CAFs have increased abundance of prometastatic proteins. (A) Functional class plot based on Cene Ontology and functional classification indicates significant changes in the TME of tumors based on CAF subtype. Each bar in the functional matrisome graph represents the total abundance of all proteins found in each functional class of matrisome proteins. Analyzed by multiple 2-tailed $t$ tests; ${ }^{*} P<0.05 ;{ }^{* *} P<0.01$.

(B) Volcano plot distribution of all detected proteins. Blue dots represent proteins detected with significantly more abundance in MCF-7/CD146 ${ }^{+}$compared with MCF-7/CD146- tumors. Red dots represent proteins detected with significantly more abundance in MCF-7/CD146- compared with MCF-7/CD146+ . (C) Representative images of tumors stained with Gomori's trichrome in the tumor center and at the tumor edge of MCF-7/CD146- and MCF-7/CD146 ${ }^{+}$ tumors. Scale bars: $200 \mathrm{~mm}$ (tumor center), $60 \mathrm{~mm}$ (tumor edge). The inset is zoomed 200\% to show blue fiber arrangement along the tumor edge. $n=6$ tumors stained for $\mathrm{CD} 146^{+}$, and $n=7$ tumors stained for CD146-

shown to increase both tumor migration and invasion and is linked to higher risk for metastasis based on its ability to align and interact directly with collagen, TNC $(17,18)$, and cancer cell integrins $(17$, 19). TNC has been shown to colocalize with fibers perpendicular to the tumor border (12), a structural feature associated with poor prognosis in hormone receptor-positive breast cancer. However, the contribution of TNC in $\mathrm{ER}^{+}$breast cancer metastasis formation has not been fully elucidated $(20,21)$.

CD146- CAFs promote ER ${ }^{+}$breast cancer metastasis in vivo. Several studies have shown that the metastatic process is accompanied by significant changes in abundance and organization of collagen fibers deposited within the ECM $(22,23)$. We used Gomori's trichrome stain to observe changes in collagen deposition in tumors excised (24) from the mammary fat pad in our cohort of mice (Figure 2C). Arrangement of the collagen fibrillar networks in the center of the tumors was similar between CAF subtypes. However, differences were observed along the tumor edge. For example, in tumors mixed with CD146- CAFs, the border areas contained thin fibers oriented perpendicular to the tumor edge. This was in contrast to the more parallel fibrillar arrangement along the borders of tumors mixed with CD $146^{+}$CAFs.

To better quantify these observations, we quantified sections stained with Picrosirius red under polarized light. Birefringence in the inner portion of tumors was not significantly different between CAF subtypes (Figure $3 \mathrm{~A}$ ); however, there was a significant decrease of birefringence along the tumor edges in 

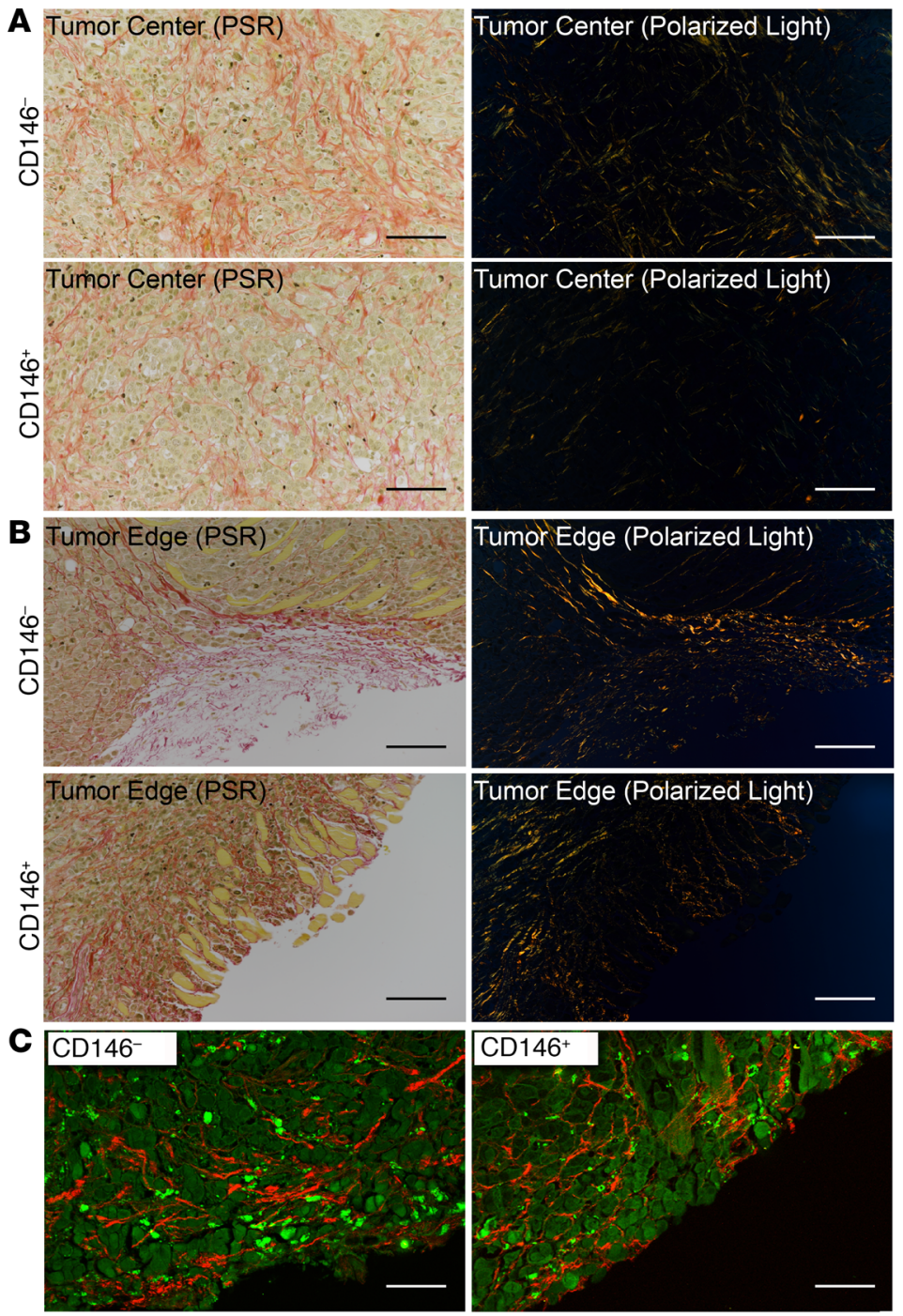
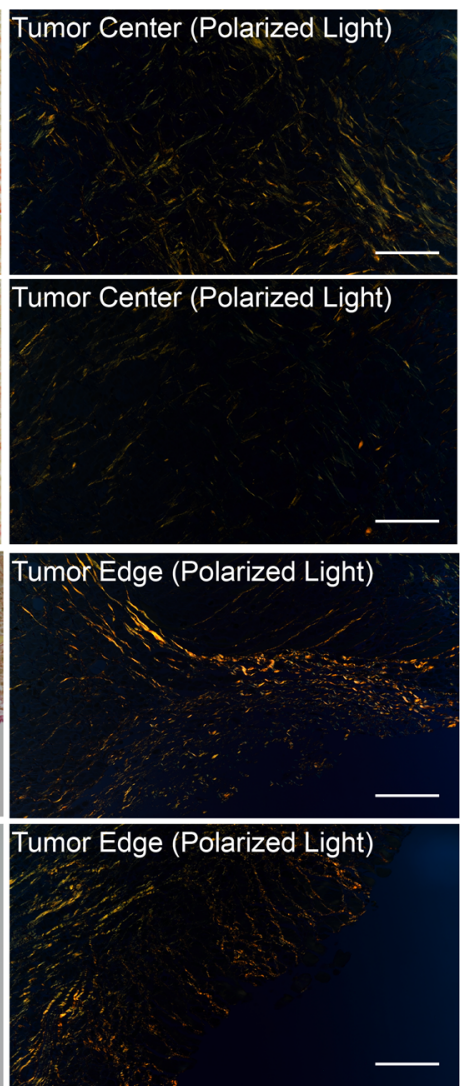

PSR Birefringence Tumor Center

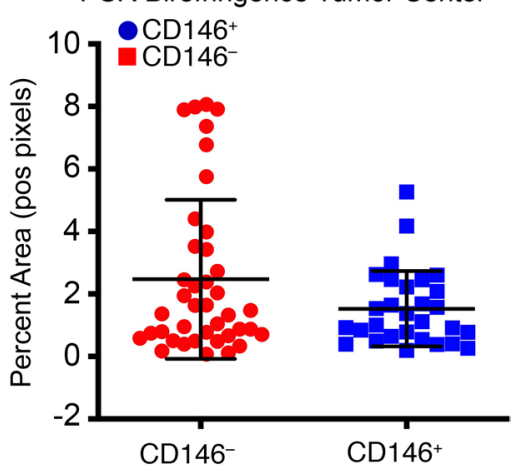

PSR Birefringence Tumor Edge
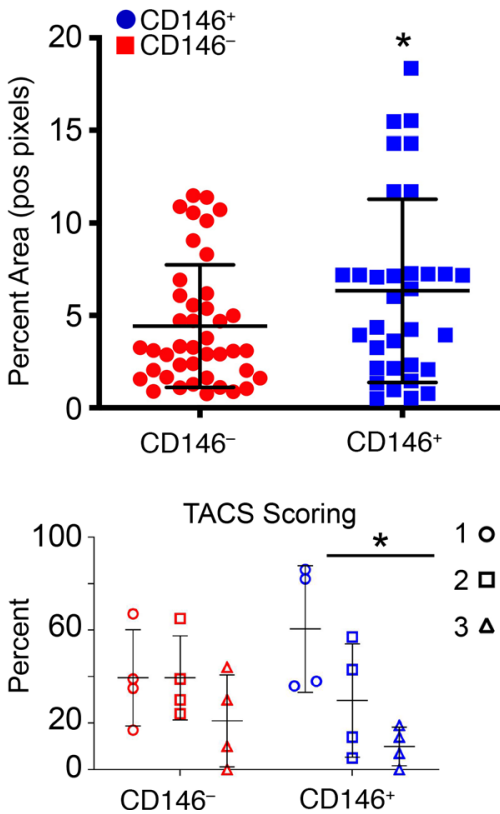

Figure 3. ECM structural organization is determined by CAF subtype. Picrosirius red (PSR) staining quantified under polarized light. (A) Birefringence in the tumor center was not significantly different between CAF subtypes. (B) Tumors influenced by CD146+ CAFs had significantly increased birefringence compared with CD146- CAFs along tumor edges. (C) Second harmonic generation (SHC) microscopy and quantified TACS scoring showed CD146 ${ }^{+}$CAF-influenced tumors had significantly more regions scored as TACS-1 compared with TACS-3. For PSR analysis at least 5 images were quantified per tumor region on $n=6$ tumors for $\mathrm{CD}_{146}{ }^{+}$and $n=7$ tumors for CD146; ; ${ }^{*}<0.05$ by unpaired 2-tailed $t$ test. For SHC analysis at least 14 images per tumor were quantified on $n=4$ CD146 and $n=4$ CD146 ${ }^{+}$tumors; ${ }^{*} P<0.05$ by 2-way ANOVA followed by Tukey's multiple-comparisons test restricted to within groups. Scale bars: 100 mM.

tumors with CD146- CAFs (Figure 3B). These results are consistent with our observations of a network of aligned fibers associated with the edges of $\mathrm{CD} 146^{-} \mathrm{CAF}$-containing tumors.

Several studies have illustrated that distinctive patterns of collagen reorganization occur during breast cancer progression, termed tumor-associated collagen signatures (TACS) (24-26). We applied SHG imaging to visualize stromal collagen and infer mechanical properties based on the appearance of wavy (i.e., relaxed) or straight (i.e., stiff) collagen fibers, followed by blinded post hoc evaluation of TACS (27-29). The TACS scale is based on scoring the abundance and orientation of collagen fibers around the tumor border, with TACS-1 defined as collagen that appears wavy, curly, and random, with increased fiber accumulation; TACS-2 defined as straightened collagen fibers that align tangentially to the tumor border; and TACS-3 defined as straight collagen fibers that align perpendicular to the tumor border. Increased TACS scores correlate with disease progression and worse patient outcomes (25). Tumors with CD146 ${ }^{+}$CAFs had significantly more TACS- 1 than TACS-3 scoring (Figure 3C), which is indicative of decreased metastatic potential, whereas tumors with CD146- CAFs had equal distribution between all TACS scores. In combination with our proteomics analysis and collagen staining results, our data indicate that CAF subtypes drive ECM remodeling in tumors. 

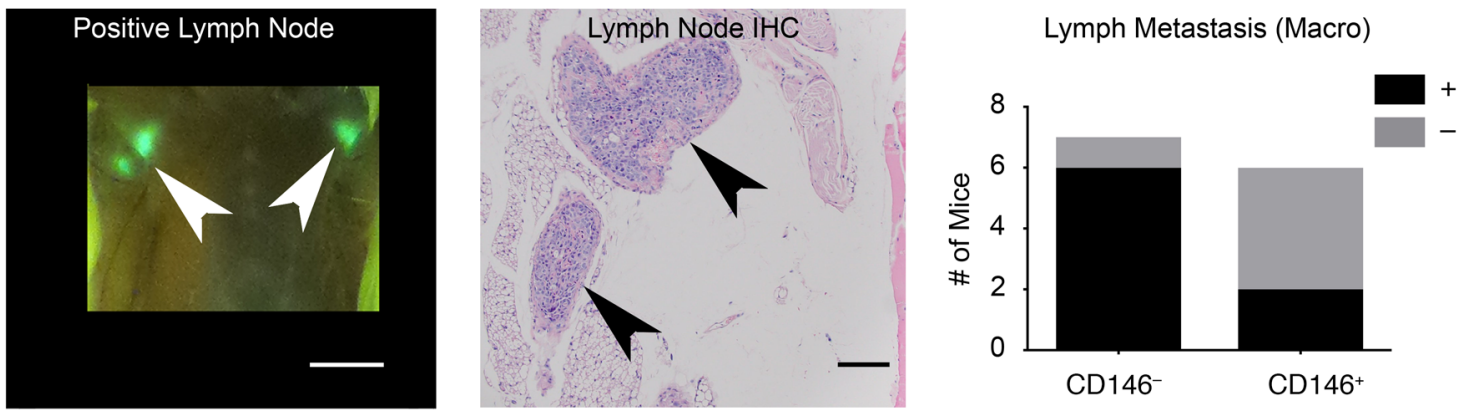

Figure 4. CD146- CAFs promote metastasis in vivo. MCF-7 tumors mixed with either CD146- or CD146+ CAFs were grown in the mammary fat pad of mice. Representative images of positive lymph nodes in whole tissue (green fluorescence) or by hematoxylin and eosin in mice with MCF-7/CD146 ${ }^{+}$or MCF-7/CD146- tumors. The presence of lymph node metastasis was not significantly different between CAF subtypes. Scale bar: $1 \mathrm{~cm}$. Contingency analysis used Fisher's exact test. $n=7$ mice with CD146- and $n=6$ mice with CD146 ${ }^{+}$.

We next wanted to determine whether these ECM changes would lead to differences in metastatic outcome. We chose MCF-7 cells for in vivo studies because this ER ${ }^{+}$cell line has a well-documented low potential for development of distant organ metastases in animal models. As expected, lymph node metastases were more common, observed in 33\% of mice with MCF-7/CD146 $\mathrm{CAF}$ tumors, compared with $86 \%$ of mice with MCF-7/CD146- CAF tumors. This difference did not reach statistical significance (Figure 4). However, $86 \%$ of mice bearing tumors mixed with $\mathrm{CD}^{146^{-}} \mathrm{CAFs}$ developed lung metastases, compared with $16 \%$ of those with CD146 $6^{+}$CAFs $(P=0.021)$ (Figure 5 , A and B).

In order to further quantify the in vivo metastatic disease burden, we paraffin embedded the whole lung of each mouse ( $n=7$ for CD146 CAF, and $n=6$ for CD146 ${ }^{+} \mathrm{CAF}$ ), stained for human specific pan-cytokeratin, and counted positive cells in all 5 lung lobes in each section (Figure 5C). In this analysis, we identified positive cells in lungs from mice with both CAF subtypes. However, mice with MCF-7/ CD146- CAF tumors had significantly more lesions per lung (69\% increase, $P<0.05$; Figure $5 \mathrm{D})$ ). Furthermore, we counted the number of cells per lesion and classified each lesion as single cell, micrometastasis ( $\leq 20$ cells), or macrometastasis ( $>20$ cells) (Figure $5 \mathrm{E}$ ). We found that the lungs from mice with CD146 CAF-bearing tumors had significantly more micrometastases compared with those with CD146 ${ }^{+} \mathrm{CAFs}$ ( $86 \%$ increase, $P<0.01$ ). We anticipate that the micrometastases would have further developed into larger lesions if given time to grow, but this study was restricted to 11 weeks' growth or primary tumors reaching $2 \mathrm{~cm}$ in any direction, at which time all samples were collected. Our data show that CD146- CAFs enhanced the metastatic potential of MCF-7 cells compared with CD146 ${ }^{+} \mathrm{CAFs}$.

We also found evidence that compared with $\mathrm{CD} 146^{+} \mathrm{CAFs}$, CD146- CAFs may encourage tumor growth, as the average volume of excised tumors was significantly different (MCF-7CD146- CAF, 570 $\pm 313 \mathrm{~mm}^{3}$ versus MCF-7/CD146 CAF, $195 \pm 99 \mathrm{~mm}^{3} ; P=0.0171$; Figure 5F). Surprisingly, when we graphed $\mathrm{CD} 146^{+} \mathrm{CAF}$-bearing tumors alone (blue dots), CD146- CAF-bearing tumors alone (red dots), or both combined, there was no correlation between final tumor volume and the number of total lesions per lung (Figure 5G). Our experimental findings are in agreement with the recent extensive analysis where metastatic outcomes and nodal involvement in a cohort of 819,647 patients with breast cancer did not correlate with primary tumor size at the time of diagnosis (30).

$T M E$-based gene signature predicts lymph node involvement in patients. Breast cancer is recognized as a systemic disease with metastatic potential regardless of the size of the primary tumor. We hypothesized that the potential of small primary tumors (T1) to develop early metastases is partly dependent on their ECM composition. Therefore, we generated a signature from our ECM proteomics data set, based on a significant difference $(P<0.05)$ between the CAF subtypes (identified as human specific or shared, Supplemental Table 2$)$. We then used data from 1009 patients with breast cancer described in The Cancer Genome Atlas (TCGA Research Network, http://www.cancer.gov/tcga) and classified primary tumors based on their ECM signature as high or low expressers. The gene signature of ECM proteins corresponding to a CD146- CAF-containing tumor was highly predictive of lymph node involvement at the time of diagnosis for both $\mathrm{T} 1$ (tumor size $<2 \mathrm{~cm}$, $P=0.015$ ) and $\mathrm{T} 2$ (tumor size between 2 and $5 \mathrm{~cm}, P=0.006$ ) tumors. High gene expression demonstrated a

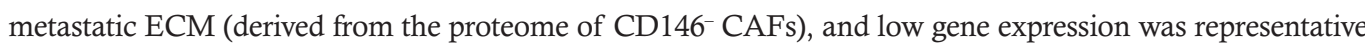
of a nonmetastatic ECM (derived from the proteome of $\mathrm{CD} 146^{+} \mathrm{CAFs}$ ) (Figure $5 \mathrm{H}$ ). The relationship did not 

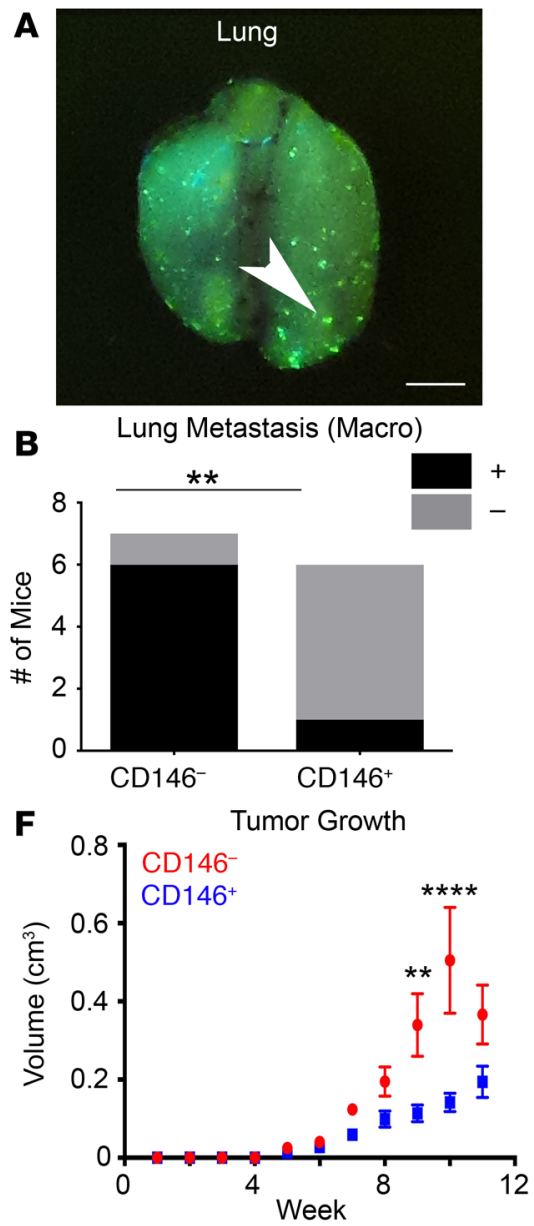
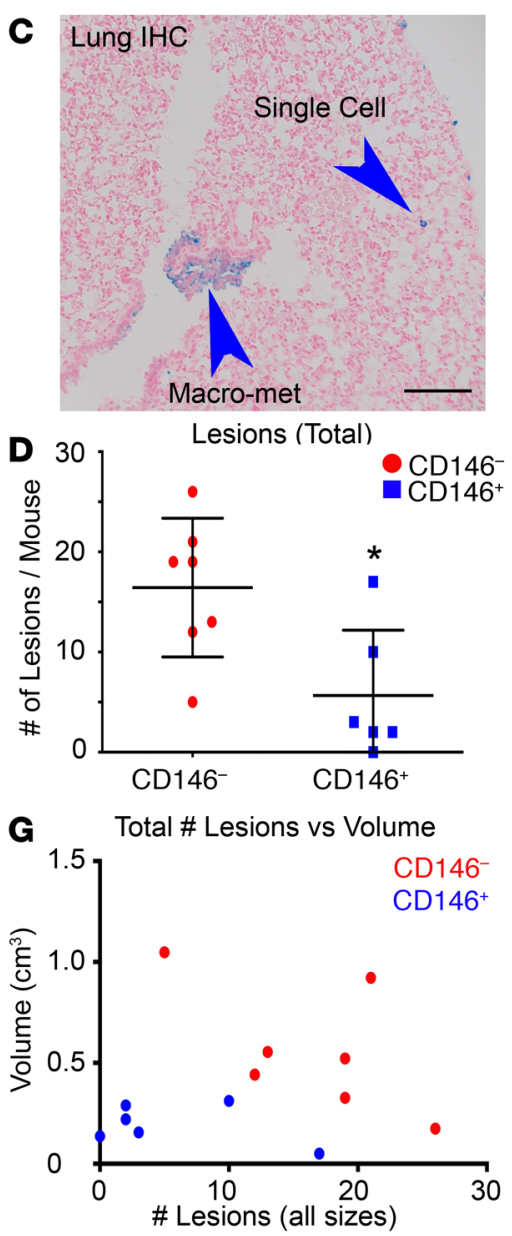
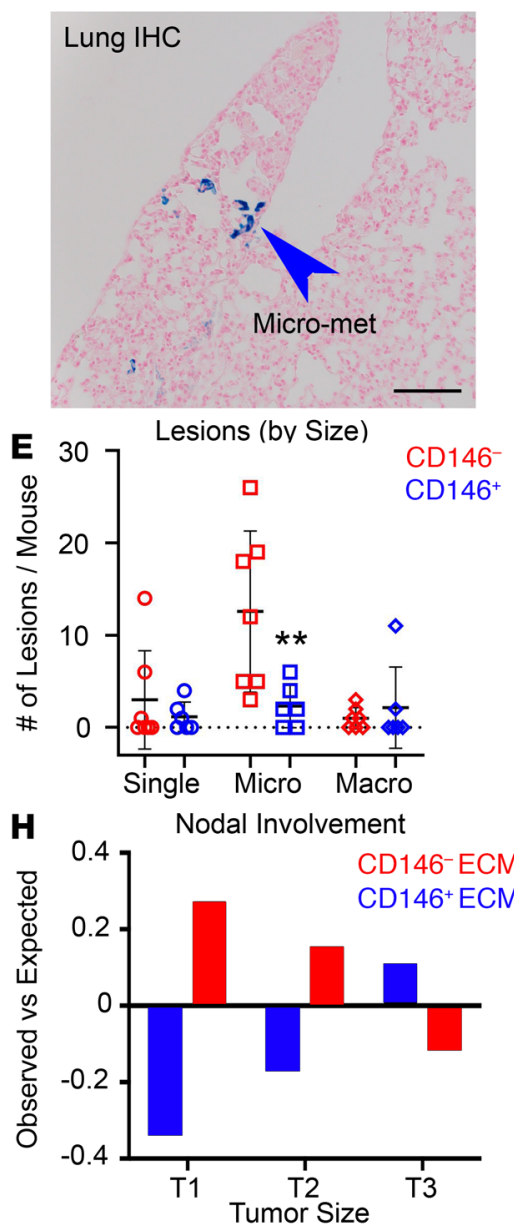

Figure 5. CD146- CAFs promote metastasis in vivo. MCF-7 tumors mixed with either CD146- or CD146+ CAFs were grown in the mammary fat pad of mice. (A and B) Representative image and quantification of metastasis to the lung of mice with MCF-7/CD146- or MCF-7/CD146 tumors in whole tissue (green fluorescence). Scale bar: 250 mm. Contingency analysis used Fisher's exact test. (C) Lesions were detected by IHC staining with human-specific pan-cytokeratin (blue) and counted in all lobes. Blue arrows show single cell, micrometastasis ( $\leq 20$ cells), or macrometastasis ( $>20$ cells). (D) Analysis of the total number of metastatic lesions showed that mice with MCF-7/CD146- tumors had significantly more lesions than those with MCF-7/CD146+ tumors. Total analysis used Mann-Whitney $U$ test. (E) Lesions were grouped according to the number of cells per lesion. Mice bearing MCF-7/CD146- tumors had significantly more micrometastases compared with MCF-7/CD146+ tumors. Lung lesion by size analysis used a 2-way ANOVA. Scale bars: 100 mm. (F) Tumors with CD146- CAFs grew significantly larger than tumors with CD146+ CAFs. Analysis was by 2-way ANOVA followed by Tukey's multiple-comparisons test. (C) The number of metastatic lung lesions did not correlate with the size of the tumor when the tumors were combined and analyzed as a total group ( $P$ $=0.6616, R^{2}=0.01806$ ) or separated by CD146- (red; $P=0.3574, R^{2}=0.1563$ ) or CD146 (blue; $P=0.4953, R^{2}=0.1231$ ) CAF influence. Correlation was tested using Pearson's correlation analysis. $(\mathbf{H})$ Gene signature based on proteomic analysis is predictive of the presence of lymph node metastasis at diagnosis in breast cancer patients with small tumors. $n=7$ mice with $\mathrm{CD} 146^{-}$, and $n=6$ mice with $\mathrm{CD}^{146+} ;{ }^{*} P<0.05 ;{ }^{* *} P<0.01 ;{ }^{* * *} P<0.0001$

hold for T3 tumors. These data support the role of ECM in development of early breast cancer metastases. To perform further mechanistic studies, we next established an in vitro spheroid assay.

CD146 CAFs promote breast cancer cell invasion. We used a 3-dimensional spheroid assay in which 600 red-tagged $\mathrm{CD} 146^{+} \mathrm{CAFs}$ or $\mathrm{CD} 146^{-} \mathrm{CAFs}$ were mixed with 3000 nuclear green-tagged MCF-7 (Figure 6A) or MDA-MB-231 (Figure 6B) tumor cells, representing 1 breast cancer cell line with low invasive potential and 1 breast cancer cell line with high invasive potential. The mixed cells formed tight spheres, with the outermost shell being composed mostly of tumor cells and the inner area being generally divided into 2 components: 1 dense region of fibroblasts and a second diffuse area of tumor cells mixed with fibroblasts. Over a defined time course (21 days for MCF-7 cultures, 6 days for MDA-MB-231 cultures), CD146CAF protrusions were documented exiting the spheres while tumor cells closely followed and invaded into the surrounding matrix. Both MCF-7 and MDA-MB-231 invaded significantly more in spheroids mixed with CD146- CAFs. MCF-7 invasion was not inhibited by CD146 overexpression in CD146- CAFs (Supplemental Figure 2, A-C). To further confirm results, we used $2 \mathrm{ER}^{+}$, patient-derived breast cancer cell 
A
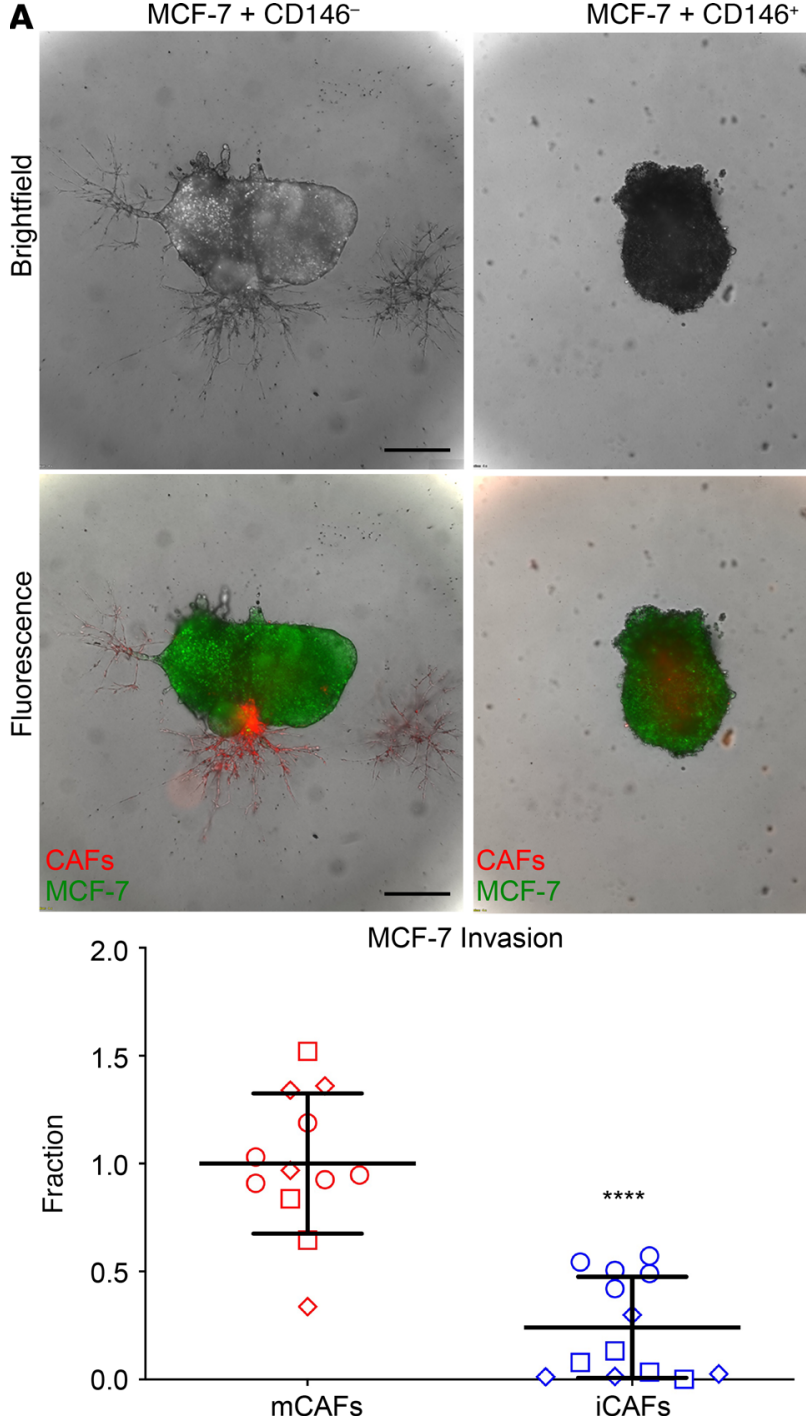

MCF-7 + CD146+
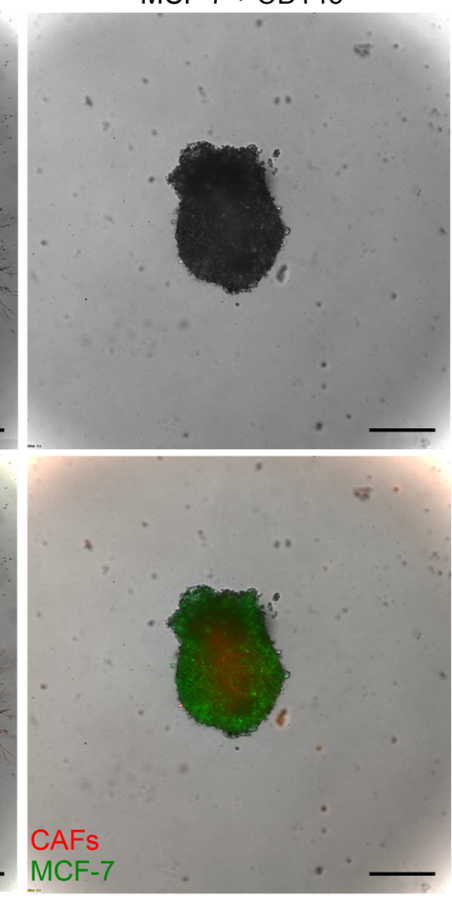

\section{m}
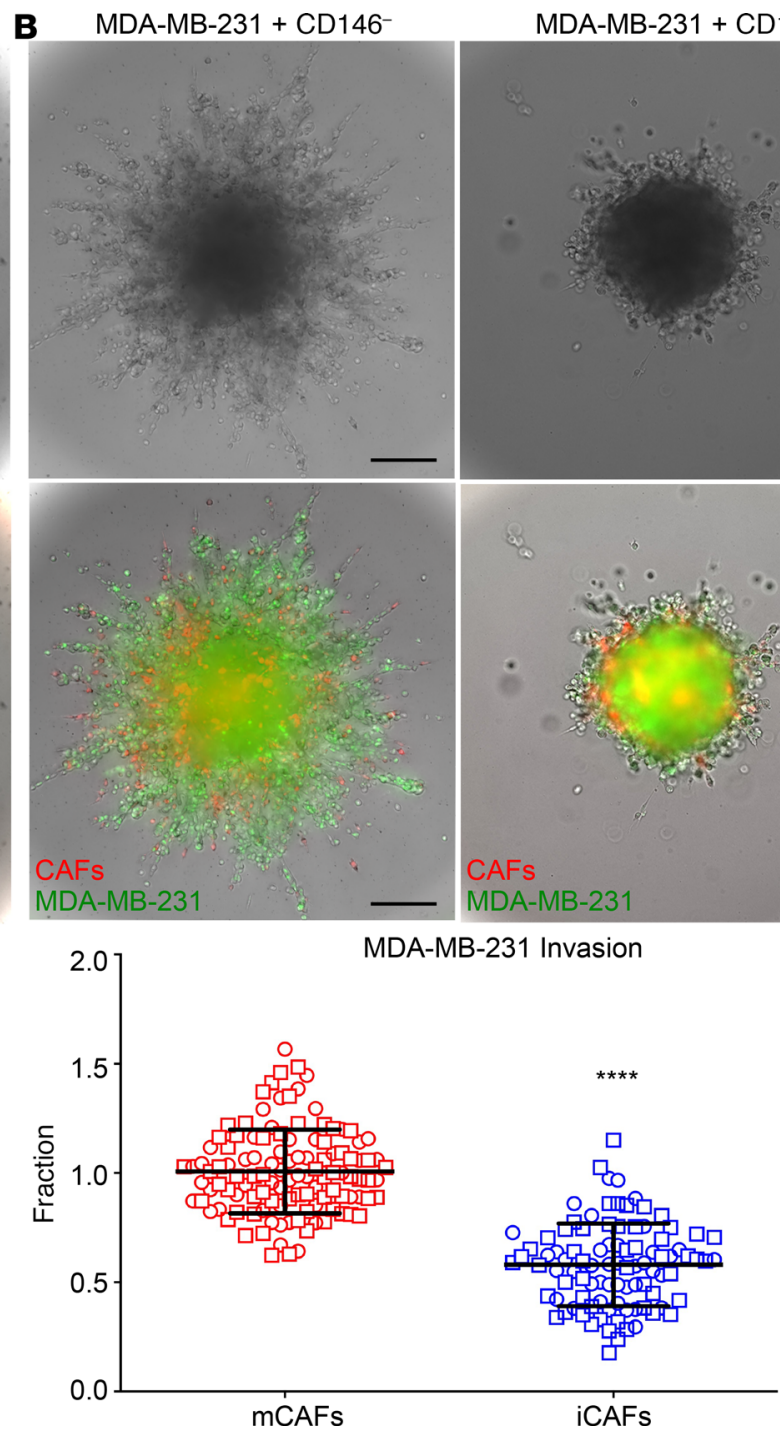

CAFs
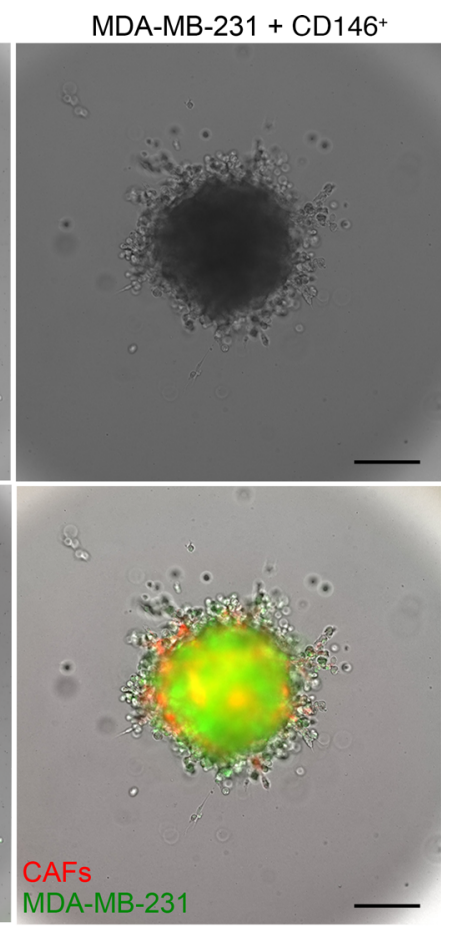

\section{.}




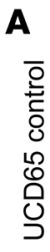
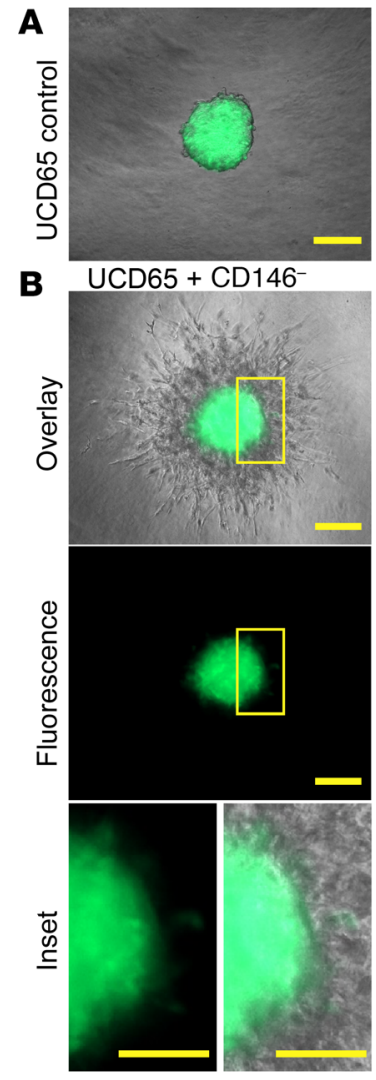

$c$
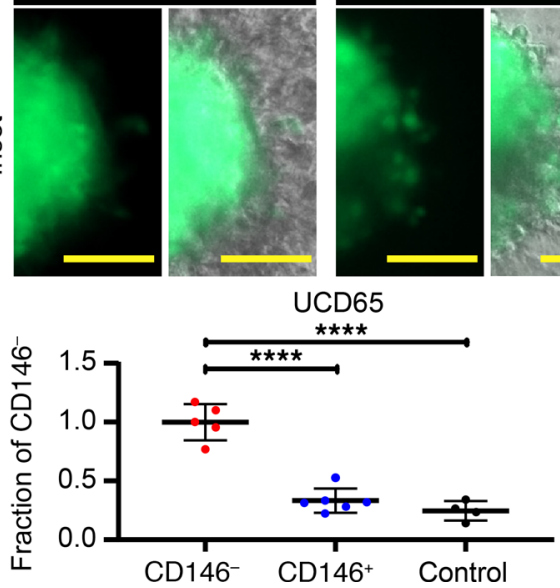
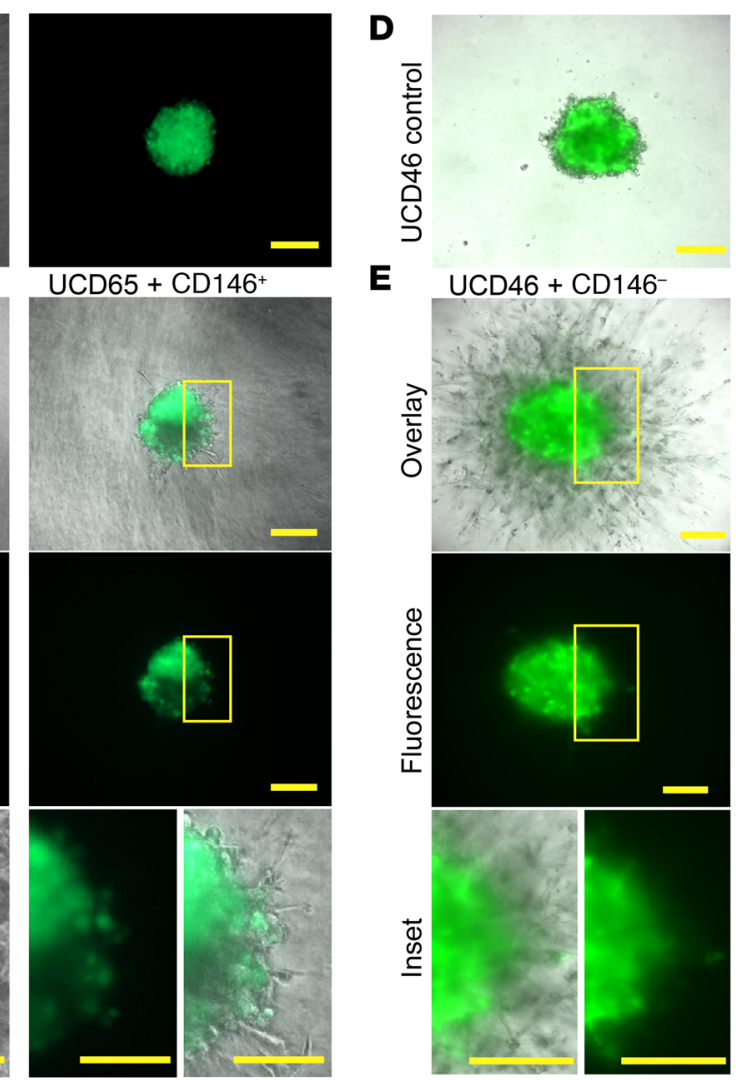

E
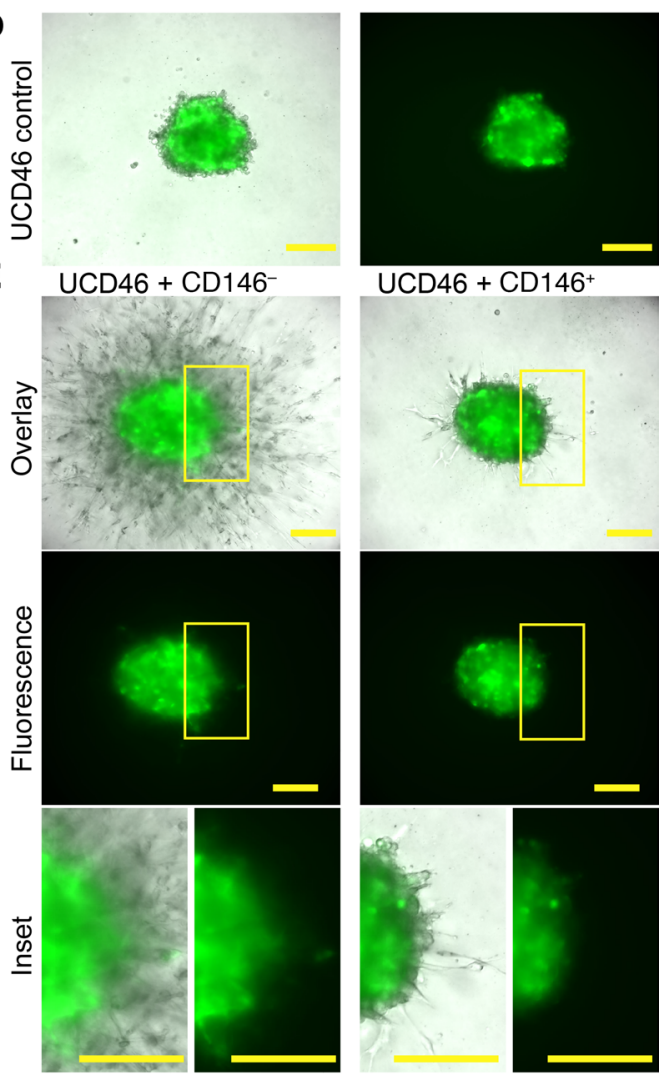

F

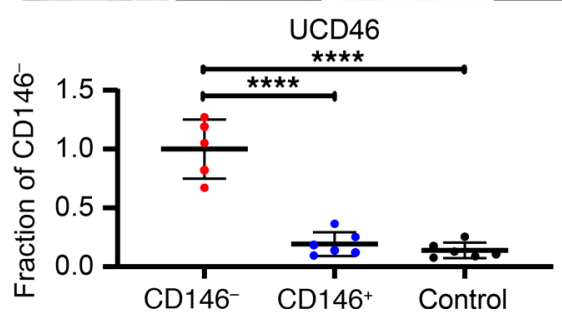

Figure 7. CD146- CAFs, but not CD146+ CAFs, promote invasion of ER+, patient-derived breast cancer cell lines UCD46 and UCD65. Representative image series showing minimal invasion for (A and B) the patient-derived UCD65 ER+ cancer cell line in spheroid cocultures. UCD65 cells were GFP labeled and cultured with unlabeled CD146- or CD146+ CAFs. Insets show zoomed-in regions demonstrating areas of invasion, with arrows pointing to representative invasive cells. (C) Quantification of invasion showing significantly increased invasion by UCD65 cells in spheroid cocultures with CD146- CAFs compared with cocultures alone or with CD146+ CAFs. (D-F) Repeat experiment shown in $\mathbf{A}$ and $\mathbf{B}$ using GFP-labeled ER ${ }^{+}$, patient-derived breast cancer cell line UCD46. $n=4-6$ spheroids quantified per group. Scale bars: $200 \mathrm{~mm}$. Ordinary 1-way ANOVA followed by Dunnett's multiple-comparisons test; ${ }^{* * * *} P<0.0001$.

CUB domain-containing protein 1 (CDCP1), which specifically marks CD146- CAFs; TNC; and nuclear fast red to demonstrate the presence of $\mathrm{CD} 146^{+} \mathrm{CAFs}$ (Supplemental Figure 3, B and C). As expected, TNC staining in these cultures was strictly confined to areas populated by CD146- CAFs. We next generated 3 CD146- CAF cell lines expressing shRNAs against TNC. Quantitative PCR and Western blot analysis confirmed between $40 \%$ and $80 \%$ loss of TNC expression in the 3 cell lines compared with the control line, which expressed shRNA against GFP (GFP was not expressed in the CD146- CAF cell line; Supplemental Figure 3D). In spheroids grown under serum-starved conditions ( $0.5 \% \mathrm{FBS})$, invasion of MCF-7 and MDAMB-231 cells was significantly inhibited by the loss of TNC expression in CD146- CAFs (Figure 8, A and B). The decrease in MCF-7 spheroid cocultures was by an average of $27 \%$, or $58 \%$ when analyzing only shRNAs TNC88 and TNC88b. For MDA-MB-231 spheroid cocultures, the average decrease in invasion was 19\%.

TNC has been shown to activate the extracellular signal-regulated kinase (ERK) pathway (32). We used IHC to stain spheroid cocultures, which were generated using CD146- CAFs expressing either control shRNA or shRNA against TNC, for phosphorylated extracellular signal-regulated kinase (p-ERK) and TNC. The invasion fronts of spheroids generated with shRNA control CD146- CAFs had significantly increased expression of TNC and p-ERK compared with spheroids generated with TNC shRNA 
A

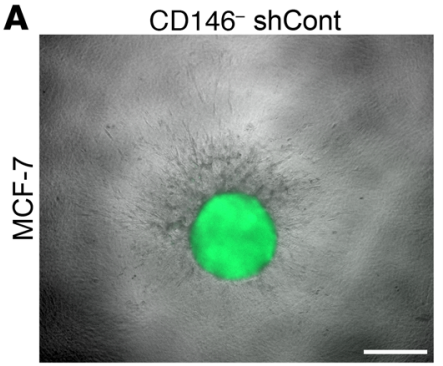

B

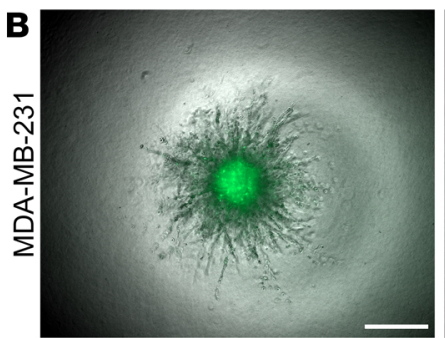

CD146- $\operatorname{shTNC88}$
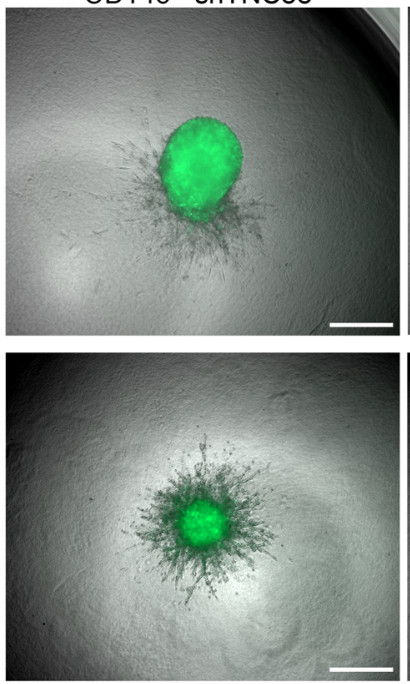

CD146- shTNC88b
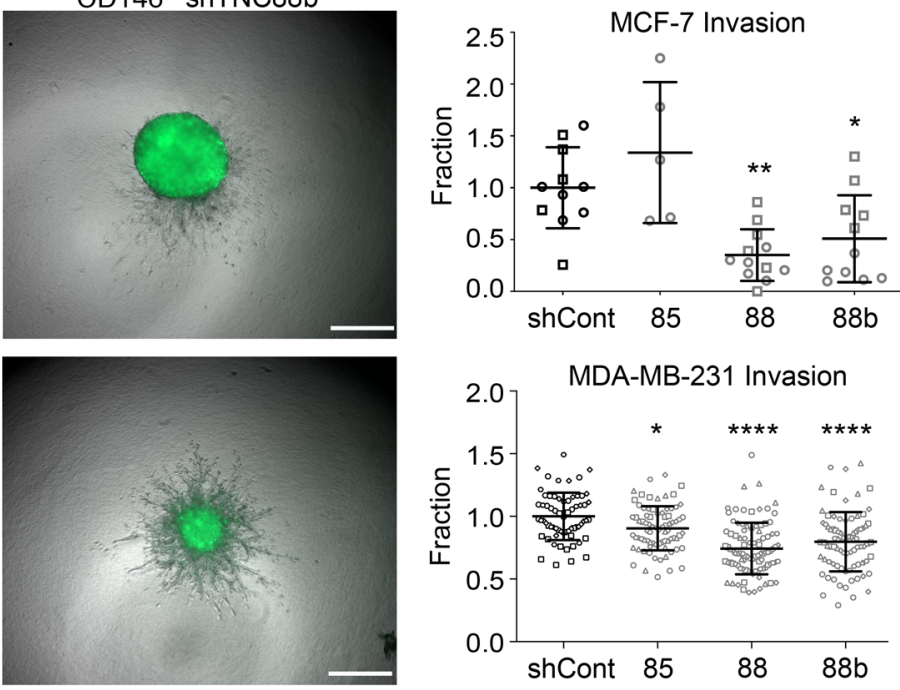

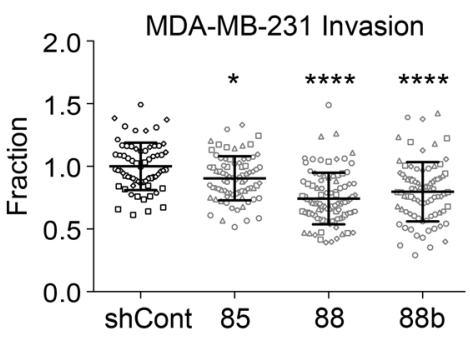

Figure 8. Loss of TNC expression decreases breast cancer cell invasion. (A) MCF-7 or (B) MDA-MB-231 spheroids formed in serum-starved conditions (0.5\% FBS) with CD146- CAFs expressing control shRNA (shCont) had significantly more invasion than spheroids with shRNA against TNC (85, 88, 88b). Experiments were individually repeated twice for MCF-7 and 5 times for MDA-MB-231. Symbols with the same shape represent replicates within the same experiment; symbols with different shapes represent repeated experiments. Scale bars: 500 mm. ${ }^{*} P<0.05$; ${ }^{* *} P<0.01$; ${ }^{* * * *} P<0.0001$; spheroids were analyzed by ordinary 1-way ANOVA followed by Dunnett's multiple-comparisons test of each column mean with the mean of shCont.

CD146- CAFs (Figure 9 and Supplemental Figure 4). Taken together, these data suggest that loss of TNC expression leads to decreased ERK signaling and significantly decreased invasion of breast cancer cells.

Loss of EGFR signaling significantly decreases spheroid invasion. TNC contains 14.5 EGF-like repeats and is a low-affinity EGFR ligand (33). Activation of EGFR is linked to increased invasion and tumor metastasis (34). The positive correlation between TNC expression and increased p-ERK, which is a common downstream effector protein of the EGFR pathway (35), suggested that EGFR signaling was a component of our invasion phenotype. When spheroid cocultures were generated in serum-starved conditions with nuclear GFP-tagged MCF-7 or MDA-MB-231 breast cancer cells, both cancer cell types were significantly less invasive with $\mathrm{CD}_{146^{+}}$CAFs compared with CD146- CAFs ( $89 \%$ and 47\% decreases, respectively; Figure 10, A and B). These data suggest that invasion in spheroid cocultures with CD146 ${ }^{+}$CAFs requires components supplied by the serum that can be compensated for in the spheroid cocultures generated with CD146- CAFs. To test whether invasion in the serum-starved spheroid cocultures with CD146- CAFs required EGFR signaling, we treated them with gefitinib, an EGFR inhibitor. Because of the loss of signaling pathways dependent on cell-to-cell interactions that might support the invasion phenotype, spheroids consisting solely of tumor cells or CAFs were not generated for treatment with gefitinib. Addition of $5 \mu \mathrm{M}$ gefitinib resulted in decreased invasion in MCF-7/CD146- CAF spheroid cocultures by $63 \%$ and by $18 \%$ in MDA-MB-231 CD146- CAF spheroid cocultures (Figure 10, A and B). These data demonstrate an important role for the EGFR signaling pathway in the invasion phenotype of the spheroid cocultures.

\section{Discussion}

Although it is widely accepted that TME remodeling is an enabling characteristic in breast cancer progression and treatment resistance, the generation of in vivo models and quantitative techniques capable of assessing the role of $\mathrm{TME}$ components in metastatic, $\mathrm{ER}^{+}$breast cancer has been the limiting factor. Historically, promising preclinical testing of drugs, such as EGFR inhibitors, designed to treat metastatic breast cancer all too often disappointed in the clinic. Many novel targeting agents, including immune checkpoint inhibitors, are clearly dependent on TME and by extension on ECM composition. Our data reinforce the idea that TME remodeling is closely tied to metastatic progression and that we have a need to develop a deeper understanding of the interplay between tumor cells, CAFs, immune cells, and the signaling changes pursuant to these interactions.

For $\mathrm{ER}^{+}$breast cancer in particular, one of the impediments to studying the TME is a lack of metastatic models that recapitulate natural disease evolution from the orthotopic site to distant organs (36). Through the use of $\mathrm{CD} 146^{+} \mathrm{CAFs}$ or CD146- CAFs, we have been able to develop a model that consistently forms 
A
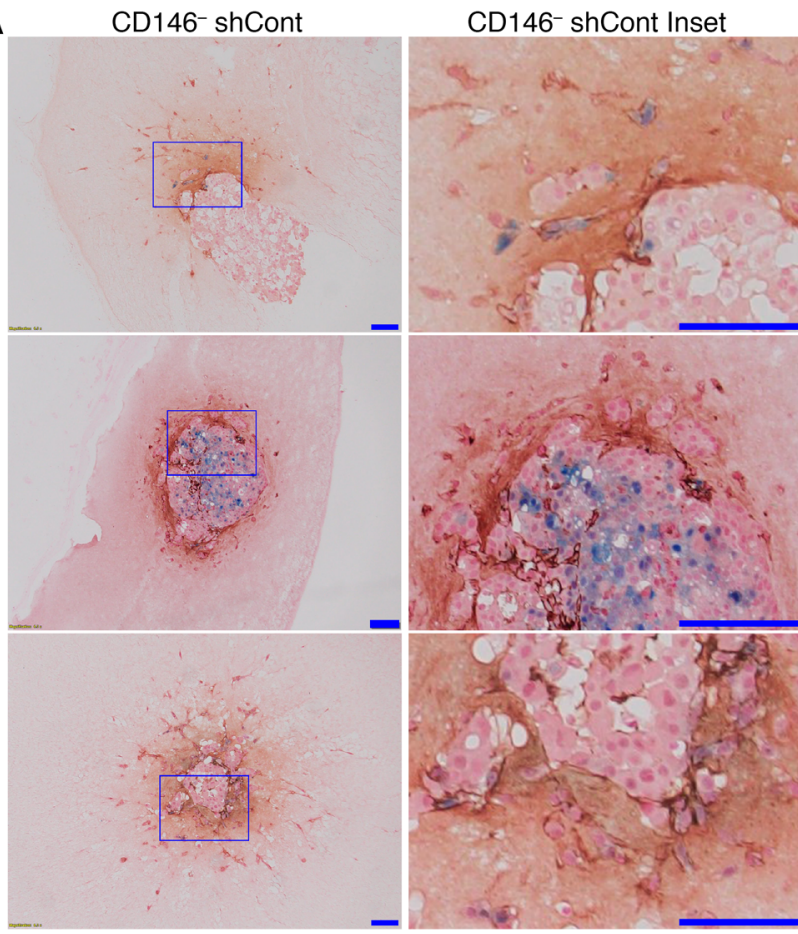

B

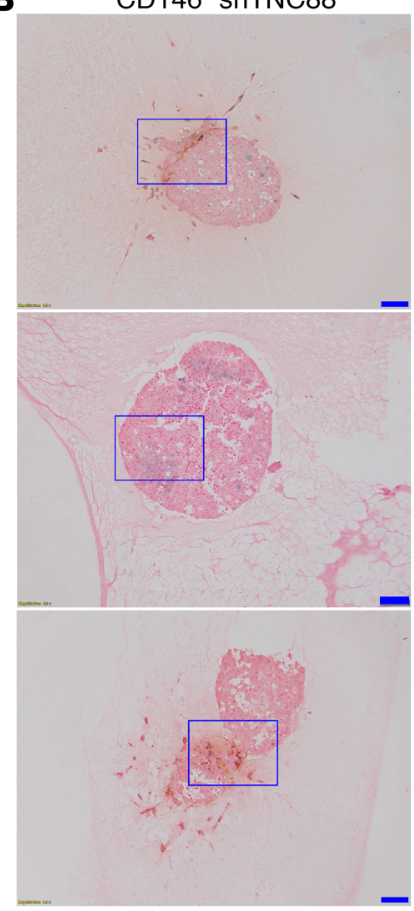

CD146- ${ }^{-}$ShTNC88 Inset

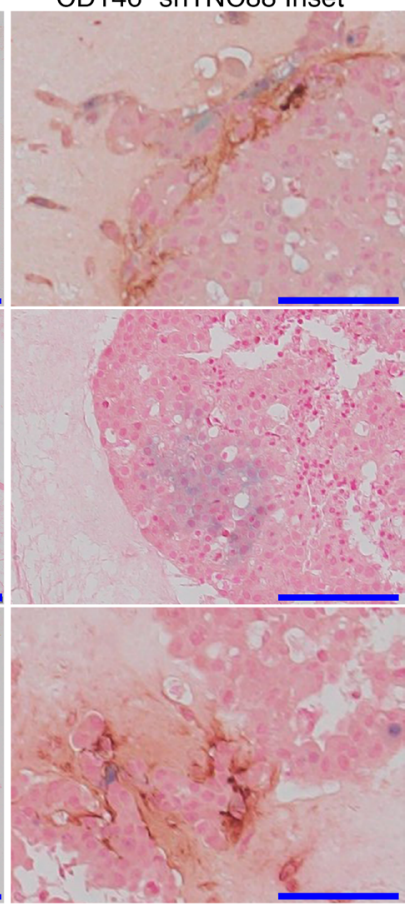

\section{C}

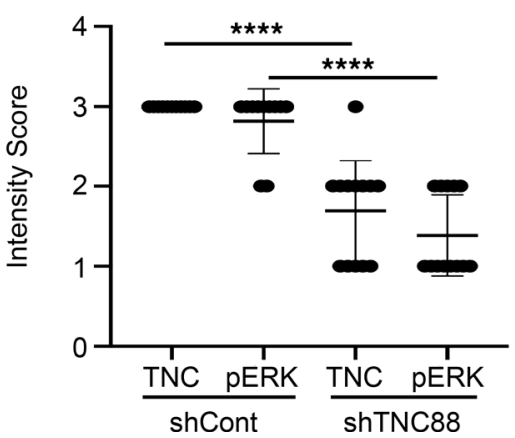

Figure 9. MCF-7 spheroid invasion fronts expressing TNC have significantly more p-ERK expression. MCF-7 spheroids were cocultured with CD146- CAFs (HS5) expressing (A) control shRNA (shCont) or (B) shRNA against TNC (shTNC88). IHC for p-ERK (pERK, blue) and TNC (brown) in 3 representative spheroids and counterstained with nuclear fast red. Insets are marked by a blue box. (c) Intensity scores on a scale of 1 to 3 (1, minimal staining; 2, moderate staining; 3 , intense staining) for p-ERK and TNC. Per spheroid 4-6 serial images were scored, with $n=3$ spheroids per group. Scale bars: $100 \mathrm{~mm} .{ }^{* * *} P<0.0001$; statistics were completed using an ordinary 1-way ANOVA followed by Tukey's multiple-comparisons test.

metastasis from the orthotopic site and has utility for comparative analysis of the TME. Our model produced increased macrometastases when tumors were influenced by CD146- CAFs compared with CD146 CAFs and similar numbers of micrometastases. Further investigation is required to determine whether the micrometastases in tumors influenced by $\mathrm{CD} 146^{+} \mathrm{CAFs}$ have the appropriate stimuli to further develop into macrometastases over time. Our model reinforces previous findings that the type of collagen deposited in the TME along with its organization around the tumor boundary are indicators of prognosis in an $\mathrm{ER}^{+}$ breast tumor. In addition, one of the unique aspects of our study is documentation of the ability to go beyond histologic description and use precise quantification of ECM components, including the ability to identify human versus host contributions. We show how consideration of TME components is predictive of lymph node involvement at the time of diagnosis in early-stage breast tumors. These findings are independent of the clinically used IHC and genomic assays focusing on receptor status and proliferative index in hormone receptor-positive breast cancer. Further refinement of this signature in a manner that also correlates with recurrence rates would add new information that may benefit patients and physicians who are weighing treatment options and deciding on the intensity of the treatment plan.

Development of metastatic disease in $\mathrm{ER}^{+}$breast cancer is commonly associated with increased EGFR signaling (37). Our in vitro results support a role for EGFR signaling in breast cancer metastasis and showed that the type of CAF present in the TME determined involvement of EGFR-mediated invasion. We also found that loss of TNC expression specifically in the CAF population resulted in decreased invasion, which correlated with decreased p-ERK expression, suggesting that TNC activates EGFR and partly mediates 
A

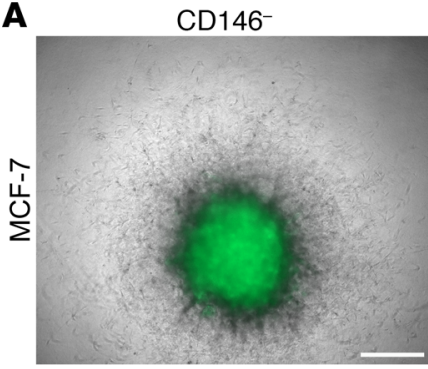

B

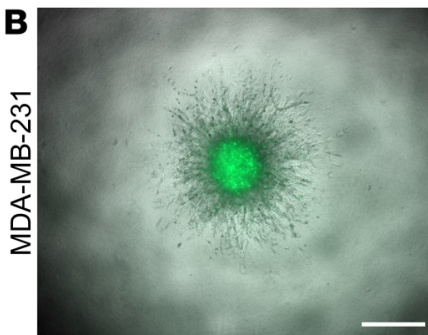

CD146
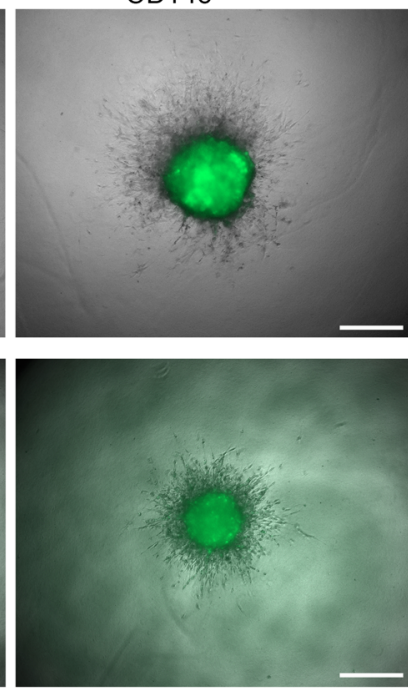
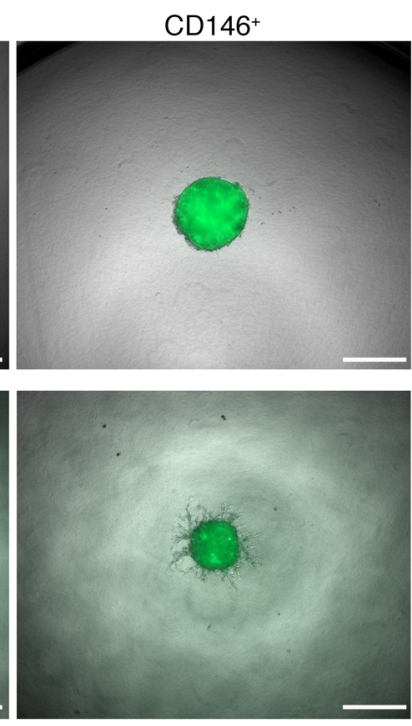
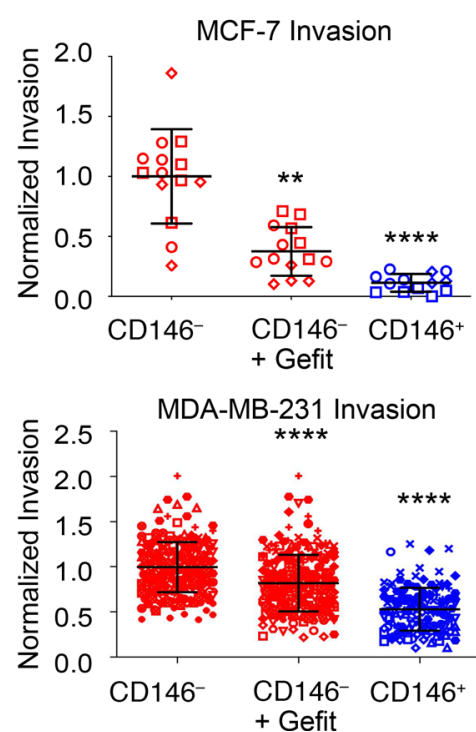

Figure 10. Loss of EGFR signaling decreases breast cancer cell invasion. (A) Compared with MCF-7 or (B) MDA-MB-231 spheroids mixed with CD146- CAFs, spheroids with $\mathrm{CD}_{146}{ }^{+}$CAFs or treated with the EGFR inhibitor gefitinib (Gefit) were significantly less invasive. Scale bars: $500 \mathrm{~mm}$. ${ }^{* *} P<0.01 ;{ }^{* * *} P<$ 0.0001 ; spheroids were analyzed with 1-way ANOVA followed by Tukey's multiple-comparisons test. All experiments were repeated 3 times. Symbols with the same shape represent replicates within the same experiment; symbols with different shapes represent repeated experiments.

an invasive phenotype in spheroid cultures. Although more studies are required to directly link the invasion phenotype with direct EGFR activation by TNC, our results recapitulate previous reports correlating increased TNC expression to higher risk for invasive breast cancer and several other cancer types (38-42). Interestingly, in a cohort of 1286 primary $\mathrm{ER}^{+}$breast tumors, TNC expression was also linked to tamoxifen resistance (20). We previously showed that influence of CD146- CAFs leads to tamoxifen resistance, which potentially places TNC as a central player in the development of endocrine resistance as well as metastatic progression of hormone receptor-positive breast cancer. TNC is a highly reactive TME protein and can mediate intracellular signaling in tumor cells through interactions with fibronectin and other cell integrins (43). Although our in vitro results suggest that interactions between EGFR and TNC represent an important axis in the invasion phenotype, the proteomic TME profile of tumors with CD146- CAFs expressed several proteins capable of governing EGFR activation, which indicates a complex in vivo signaling environment that is likely governed by more than TNC/ERK/EGFR interactions.

Our data highlight the importance of placing the TME in context with other known drivers of breast cancer progression, such as endocrine receptor status. Taken together, this work provides a model to study the role of the TME in development of breast cancer metastasis and supports the need to consider and target cell-matrix interactions in future drug development.

\section{Methods}

\section{Cell culture}

The human MCF-7 (p53 WT, ER ${ }^{+}$, luminal subtype) and MDA-MB-231 (triple negative, EGFR ${ }^{+}$) breast cancer cell lines and the human stromal HS5 fibroblast cell line were cultured in DMEM from Corning (MT10013CVEA) supplemented with $10 \%$ fetal calf serum, L-glutamine, and HEPES buffer at $37^{\circ} \mathrm{C}$ with $5 \% \mathrm{CO}_{2} / 95 \%$ atmospheric air. The human stromal HS27A fibroblast cell line was cultured in RPMI 1640 basal medium (Corning, MT10040VCEA) supplemented with 10\% fetal calf serum, L-glutamine, and HEPES buffer at $37^{\circ} \mathrm{C}$ with $5 \% \mathrm{CO}_{2} / 95 \%$ atmospheric air. In all coculture experiments, the base medium used was MEM. HS5 cell lines expressing shRNAs against TNC or the scrambled control were produced using standard viral transduction methods. Knockdown efficiency was determined by real-time quantitative PCR. All cell lines were authenticated by short tandem repeat profile testing in May 2016. All cell lines were validated to be mycoplasma free before use in any in vitro or in vivo experiments using the Universal Mycoplasma Detection Kit from the American Type Culture Collection. 
We previously validated the HS5 and HS27A fibroblasts as genetically akin to primary human fibroblasts, which we isolated from $\mathrm{ER}^{+}$breast cancer patient tissue (5). However, unlike the HS27A and HS5 fibroblast cell lines, patient CAFs are passage limited and difficult to expand in sufficient quantities for multiple in vitro studies and for large in vivo studies. Given these limitations and our previous validation, we chose to use HS5 and HS27A fibroblasts as a reasonable substitute for our experiments.

\section{Antibody and shRNA sources}

Lung IHC. The primary antibody to detect human tumor cells (MCF-7) was pan-cytokeratin from Biorbyt (orb386219; dilution 1:150). Secondary detection was with anti-rabbit alkaline phosphatase IgG followed by Vector Blue Alkaline Phosphatase Substrate Kit from Vector Labs (MP-5401 and SK-5300; dilutions according to the manufacturer's recommendations).

Cocultures. The primary antibody to detect MDA-MB-231 and MCF-7 cells was pan-cytokeratin from Bioss Antibodies (Bs-1712R; dilution 1:100). The primary antibody to detect HS27A cells was CD146 from Abcam (ab75769; dilution 1:100). The primary antibody to detect HS5 cells was CDCP1 from R\&D Systems (AF2666; dilution 1:40). The primary antibody to detect TNC was from Abcam (ab3970; dilution 1:100). The primary antibody to detect p-ERK was from Invitrogen, Thermo Fisher Scientific (14-9109-80; dilution 1:200). All secondaries and chromogens were used according to manufacturer recommendations and purchased from Vector Labs (MP-7401, SK-4105, MP-5405, SK-5300, MP-7402, SK-4605).

For shRNA and overexpression experiments, all vectors were purchased from the Functional Genomics Facility, a University of Colorado Cancer Center Shared Resource. Two TNC-directed shRNAs were used, TNC85 (TRCN0000230785) and TNC88 (TRCN0000230788). TNC88b is a second population of HS5 cells transduced with the TNC88b viral particles. The nontargeting control was SHC002. One MCAM (CD146) open reading frame overexpression vector was used (06567).

\section{Invasion assay}

Invasion was measured by generating single spheroids of either MDA-MB-231 or MCF-7 cell lines mixed with either HS5 (CD146- CAFs) or HS27A (CD146+ CAFs) fibroblasts. We used the Cultrex 3D Cell Invasion Assay (Trevigen) following the manufacturer's guidelines for generating spheres in a 96-well round-bottom plate. Spheroids were then transferred to a flat-bottom plate with a set layer of invasion matrix and sandwiched with a second layer of invasion matrix.

Nuclear green-tagged MCF-7 cells were mixed with uncolored CAFs, and invasion was quantified on day 19. MCF-7 invasion was analyzed with Fiji (https://fiji.sc) for corrected total cell fluorescence (CTCF) to quantify the green fluorescent signal in the invasion region (44). Briefly, invasion CTCF was measured via freeform drawing using an Intuos 2 tablet (Wacom) to define the total tumor with invasion, the main sphere body, and a nonfluorescent background sample (Supplemental Figure 5A). Invasion was calculated using the following formula: invasion CTCF $=$ (total tumor integrated density - [area of invasion $\times$ background mean]) - (spheroid integrated density - [area of spheroid $\times$ background mean]).

Invasion in spheroids generated by mixing nuclear green-tagged MDA-MB-231 with uncolored $\mathrm{CD}_{146^{+}}$or CD146- was quantified on day 6 using Zen 2.3 lite (Carl Zeiss Microscopy). A circle was drawn around the main spheroid, and length of invasion arms was measured from the most distant tumor cell per arm to the spheroid body (Supplemental Figure 5B). To validate the methods, we reanalyzed MDAMB-231 coculture spheroids using the method for MCF-7 spheroids (Supplemental Figure 5, C and D). Both methods produced highly reproducible results for the MDA-MB-231 spheroids, which provides validation for the method used to measure MCF-7 spheroids. Images for these analyses' measurements were captured on a Zeiss Axio Vert.A1 fluorescent microscope (Carl Zeiss Microscopy).

We next generated DsRed-tagged $\mathrm{CD} 146^{+}$and $\mathrm{CD} 146^{-}$using viral transduction to generate more precise images of CAF and tumor cell interactions in the mixed spheroids and for comparison with higher magnification imaging on the Zeiss Axio Vert.A1 microscope. To obtain high-magnification images without interference from the concentrated fluorescence in the sphere body, we had to close the aperture to focus ZsGreen-labeled MCF-7 cells invaded away from the main sphere body in coculture with CD146- CAFs. A single uncolored fibroblast can be seen at the tip of the invasion arm (Supplemental Figure 5E). In comparison, high-resolution live imaging of GFP-labeled MDA-MB-231 with red fluorescent protein-labeled $\mathrm{CD}_{146}{ }^{-}$(Supplemental Figure 5F) or $\mathrm{CD}^{+} 4^{+}$(Supplemental Figure 5G) CAFs similarly demonstrate the generation of invading cells in arms composed of CAFs and MDA-MB-231 cells, with several arms of the 
MDA-MB-231 CD146- spheroid culture being tipped by CAFs. High-resolution live imaging was captured using an Olympus IX83 P2ZF. Images using the 2-color model were captured on day 21 for MCF-7 CAF spheroids and on day 6 for MDA-MB-231 CAF spheroids.

\section{Animal experiments}

MCF-7 tumors labeled with ZsGreen were established by mixing $1 \times 10^{6}$ cells in Cultrex (Trevigen, 3433 00-R1) and injecting them into the mammary fat pad of NSG female mice (The Jackson Laboratory, 005557). HS27A or HS5 cells were mixed with the tumor cells at a 1:1 ratio $(n=7$ mice per stroma subtype). Tumors were allowed to grow for 11 weeks or until they reached protocol limits $(2 \mathrm{~cm}$ measured in any direction) before removal. All tumors received continuous estrogen supplementation throughout the study, as previously described (45). Tumor measurements were taken weekly throughout the duration of the experiment. Metastasis was monitored by examining excised organs with an Illumatool light source (Lightools Research) attached to an Olympus camera. Organs were collected for IHC validation of wholemount results. Lymph node tumor validation was completed by H\&E staining. Lung tumor validation was monitored by staining for human-specific pan-cytokeratin. IHC imaging was captured using an Olympus IX83 P2ZF microscope. SHG microscopy was performed using a Zeiss LSM780 confocal microscope equipped with a tunable infrared Coherent Chameleon Ultra II laser.

IHC image capture and analysis. IHC imaging for lymph and lung tumor validation was captured using an Olympus IX83 P2ZF microscope. Picrosirius red staining was captured using a Nikon Eclipse Ni equipped with a Nikon DS-Ri2 camera and a polarized light filter. SHG microscopy was performed using a Zeiss LSM780 confocal microscope equipped with a tunable infrared Coherent Chameleon Ultra II laser. IHC was quantified using Fiji (46).

\section{Proteomics analysis}

Proteomic sample preparation. Tissue samples were flash frozen in liquid nitrogen and powderized using a ceramic mortar and pestle. Tissue was dried overnight in a lyophilizer, and weighed tissue (approximately $1 \mathrm{mg}$ of each) was homogenized in freshly prepared high-salt buffer (50 mM Tris- $\mathrm{HCl}, 3 \mathrm{M} \mathrm{NaCl}, 25 \mathrm{mM}$ EDTA, $0.25 \% w / v$ CHAPS, $\mathrm{pH} 7.5$ ) containing $1 \times$ protease inhibitor (Halt Protease Inhibitor, Thermo Fisher Scientific) at a concentration of $10 \mathrm{mg} / \mathrm{mL}$. Homogenization took place in a bead beater (Bullet Blender Storm 24, Next Advance, 1-mm glass beads) for 3 minutes at $4^{\circ} \mathrm{C}$. Samples were then spun for 20 minutes at $18,000 \mathrm{~g}$ at $4^{\circ} \mathrm{C}$, and the supernatant was removed and stored as the cellular fraction. Sequential tissue extraction on the remaining pellets was performed as previously described (11).

Trypsin digestion. One hundred microliters of the cellular fraction (combined fractions 1, 2, and 3) and $200 \mu \mathrm{L}$ of fractions 4 and 5 of all samples were subsequently subjected to reduction, alkylation, and enzymatic digestion with trypsin. One hundred fmols of each stable isotope-labeled peptide (770 peptides total: 170 mouse, 188 human, 412 shared) were spiked into $100 \mu \mathrm{L}$ of sample to allow for 4 injections per sample (50 fmols ECM concatemers 1-6 per injection) $(9,47)$. A filter-aided sample preparation approach, as well as C18 cleanup, were performed as previously described (10).

LC-SRM analysis. Samples were analyzed by LC-SRM and liquid chromatography-tandem mass spectrometry as described (47). Equal volumes from each postdigestion sample were combined and injected every third run and used to monitor technical reproducibility. Skyline (version 3.7) was used for method development and to extract the ratio of endogenous light peptides to heavy internal standards from LC-SRM data for protein quantification as described (48). Limits of detection, quantification, and dynamic range were determined for each peptide as previously described (9). Final fmol values are expressed as $\mathrm{fmol} / \mathrm{mg}$ where milligrams represent milligrams of starting dry tissue weight.

Proteomic data analysis. PCA was performed using GraphPad Prism (version 8), and partial least squares-discriminant analysis was performed using MetaboAnalyst (version 3.0) with sum and range scaling normalizations (49). For proteomics analysis, 2-group comparisons were made, and we used the FDR method for multiple-measurement correction. Heatmaps were generated with Heatmapper using average linkage for clustering and Pearson for distance measurement (50).

\section{Statistics}

Statistical analysis was completed using $\mathrm{R}$ package software for the gene expression data sets and with GraphPad Prism 8 analytical software for all other experiments. For single comparisons we used unpaired 
2-tailed $t$ tests with assumptions of parametric Gaussian distribution and equal standard deviations. For multiple comparisons we used ordinary 1-way ANOVA analysis with Tukey's multiple-comparisons tests. For contingency analysis we used Fisher's exact test. Significance was set at $P<0.05$. All cell culture experiments consisted of at least $n=4$ and were repeated at least once. Our in vivo experiment consisted of $n=$ 7 animals per stromal subtype. Outliers were identified using the GraphPad Prism Outlier ROUT function with $Q=1 \%$. All data are presented as mean \pm the standard deviation.

Study approval. All animal experiments were conducted in an Association for Assessment and Accreditation of Laboratory Animal Care-accredited facility at the University of Colorado Denver under an IACUC-approved protocol.

\section{Author contributions}

$\mathrm{HMB}, \mathrm{KH}$, and $\mathrm{PK}$ designed experiments. $\mathrm{HMB}, \mathrm{ASB}, \mathrm{EK}, \mathrm{JCH}, \mathrm{ALH}$, and JFS conducted experiments. $\mathrm{HMB}, \mathrm{ASB}, \mathrm{EK}, \mathrm{AEG}, \mathrm{KH}$, and $\mathrm{PK}$ analyzed results. $\mathrm{HMB}, \mathrm{DMC}, \mathrm{PO}, \mathrm{KBH}, \mathrm{CAS}, \mathrm{KH}$, and PK wrote and edited the manuscript. $\mathrm{HMB}, \mathrm{KH}$, and $\mathrm{PK}$ conceived the study. PK supervised the study.

\section{Acknowledgments}

This work was supported in part by NIH grants R01CA205044 (to PK), 5R01CA140985 (to CAS), and P30CA046934 (supporting the University of Colorado Cancer Center); the Breast Cancer Research Foundation (to KBH and CAS); and Veterans Affairs grant 1KBX00002929 (to PO). We would like to acknowledge the following core facilities and individuals for their support: the University of Colorado Denver Animal Core Facility for assistance with all animal experiments, the laboratory of MM Pillai for providing HS27A and HS5 cell lines, the laboratory of CAS for providing MCF-7 and MDA-MB-231 breast cancer cell lines, the Biochemistry and Molecular Genetics High-Throughput Sequencing Core at the University of Colorado Denver's School of Medicine, and the Advanced Light Microscopy Core Facility at the University of Colorado Denver. We thank Veronica Wessells for assistance with tissue processing.

Address correspondence to: Peter Kabos or Kirk Hansen, University of Colorado Denver, 12801 East 17th Avenue, RC1 South, MS 8117 (PK) or MS 8101 (KH), Aurora, Colorado 80045, USA. Phone: 303.724.4690; Email: peter.kabos@ucdenver.edu (PK). Phone: 303.724.5544; Email: kirk.hansen@ucdenver.edu (KH).

1. Manou D, et al. The complex interplay between extracellular matrix and cells in tissues. Methods Mol Biol. 2019;1952:1-20.

2. Kim SH, Turnbull J, Guimond S. Extracellular matrix and cell signalling: the dynamic cooperation of integrin, proteoglycan and growth factor receptor. J Endocrinol. 2011;209(2):139-151.

3. Yang Z, Ni W, Cui C, Fang L, Xuan Y. Tenascin C is a prognostic determinant and potential cancer-associated fibroblasts marker for breast ductal carcinoma. Exp Mol Pathol. 2017;102(2):262-267.

4. Hancox RA, et al. Tumour-associated tenascin-C isoforms promote breast cancer cell invasion and growth by matrix metalloproteinase-dependent and independent mechanisms. Breast Cancer Res. 2009;11(2):R24.

5. Brechbuhl HM, et al. Fibroblast subtypes regulate responsiveness of luminal breast cancer to estrogen. Clin Cancer Res. 2017;23(7):1710-1721.

6. Marsh T, Pietras K, McAllister SS. Fibroblasts as architects of cancer pathogenesis. Biochim Biophys Acta. 2013;1832(7):1070-1078.

7. Sotgia F, et al. Caveolin-1-/- null mammary stromal fibroblasts share characteristics with human breast cancer-associated fibroblasts. Am J Pathol. 2009;174(3):746-761.

8. Shan-Wei W, Kan-Lun X, Shu-Qin R, Li-Li Z, Li-Rong C. Overexpression of caveolin-1 in cancer-associated fibroblasts predicts good outcome in breast cancer. Breast Care (Basel). 2012;7(6):477-483.

9. Hill RC, Calle EA, Dzieciatkowska M, Niklason LE, Hansen KC. Quantification of extracellular matrix proteins from a rat lung scaffold to provide a molecular readout for tissue engineering. Mol Cell Proteomics. 2015;14(4):961-973.

10. Goddard ET, et al. Quantitative extracellular matrix proteomics to study mammary and liver tissue microenvironments. Int $J$ Biochem Cell Biol. 2016;81(pt A):223-232.

11. Barrett AS, et al. Hydroxylamine chemical digestion for insoluble extracellular matrix characterization. $J$ Proteome Res. 2017;16(11):4177-4184.

12. Tomko LA, et al. Targeted matrisome analysis identifies thrombospondin-2 and tenascin-C in aligned collagen stroma from invasive breast carcinoma. Sci Rep. 2018;8(1):12941.

13. Huang G, Ge G, Izzi V, Greenspan DS. $\alpha 3$ Chains of type V collagen regulate breast tumour growth via glypican-1. Nat Commun. 2017;8:14351.

14. Jin R, et al. The highly expressed COL4A1 genes contributes to the proliferation and migration of the invasive ductal carcinomas. Oncotarget. 2017;8(35):58172-58183.

15. Nissen NI, Karsdal M, Willumsen N. Collagens and Cancer associated fibroblasts in the reactive stroma and its relation to Cancer biology. J Exp Clin Cancer Res. 2019;38(1):115.

16. Wang K, et al. Breast cancer cells alter the dynamics of stromal fibronectin-collagen interactions. Matrix Biol. 2017;60-61:86-95. 
17. Erdogan B, et al. Cancer-associated fibroblasts promote directional cancer cell migration by aligning fibronectin. $J$ Cell Biol. 2017;216(11):3799-3816.

18. Soikkeli J, et al. Metastatic outgrowth encompasses COL-I, FN1, and POSTN up-regulation and assembly to fibrillar networks regulating cell adhesion, migration, and growth. Am J Pathol. 2010;177(1):387-403.

19. Kenny HA, et al. Mesothelial cells promote early ovarian cancer metastasis through fibronectin secretion. J Clin Invest. 2014;124(10):4614-4628.

20. Helleman J, et al. Association of an extracellular matrix gene cluster with breast cancer prognosis and endocrine therapy response. Clin Cancer Res. 2008;14(17):5555-5564.

21. Lowy CM, Oskarsson T. Tenascin C in metastasis: A view from the invasive front. Cell Adh Migr. 2015;9(1-2):112-124.

22. Egeblad M, Rasch MG, Weaver VM. Dynamic interplay between the collagen scaffold and tumor evolution. Curr Opin Cell Biol. 2010;22(5):697-706

23. Perumal S, Antipova O, Orgel JP. Collagen fibril architecture, domain organization, and triple-helical conformation govern its proteolysis. Proc Natl Acad Sci USA. 2008;105(8):2824-2829.

24. Provenzano PP, et al. Collagen density promotes mammary tumor initiation and progression. BMC Med. $2008 ; 6: 11$.

25. Conklin MW, et al. Aligned collagen is a prognostic signature for survival in human breast carcinoma. Am J Pathol. 2011;178(3):1221-1232.

26. Riching KM, et al. 3D collagen alignment limits protrusions to enhance breast cancer cell persistence. Biophys J. 2014;107(11):2546-2558.

27. Acerbi I, et al. Human breast cancer invasion and aggression correlates with ECM stiffening and immune cell infiltration. Integr Biol (Camb). 2015;7(10):1120-1134.

28. Butcher DT, Alliston T, Weaver VM. A tense situation: forcing tumour progression. Nat Rev Cancer. 2009;9(2):108-122.

29. Levental KR, et al. Matrix crosslinking forces tumor progression by enhancing integrin signaling. Cell. 2009;139(5):891-906.

30. Sopik V, Narod SA. The relationship between tumour size, nodal status and distant metastases: on the origins of breast cancer. Breast Cancer Res Treat. 2018;170(3):647-656.

31. Rutnam ZJ, Yang BB. The non-coding 3' UTR of CD44 induces metastasis by regulating extracellular matrix functions. $J$ Cell Sci. 2012;125(Pt 8):2075-2085.

32. Ruiz C, et al. Growth promoting signaling by tenascin-C [corrected]. Cancer Res. 2004;64(20):7377-7385.

33. Swindle CS, et al. Epidermal growth factor (EGF)-like repeats of human tenascin-C as ligands for EGF receptor. $J$ Cell Biol. 2001;154(2):459-468.

34. Lu Z, Jiang G, Blume-Jensen P, Hunter T. Epidermal growth factor-induced tumor cell invasion and metastasis initiated by dephosphorylation and downregulation of focal adhesion kinase. Mol Cell Biol. 2001;21(12):4016-4031.

35. Wee P, Wang Z. Epidermal growth factor receptor cell proliferation signaling pathways. Cancers (Basel). $2017 ; 9(5):$ E52.

36. Fantozzi A, Christofori G. Mouse models of breast cancer metastasis. Breast Cancer Res. 2006;8(4):212.

37. Foley J, et al. EGFR signaling in breast cancer: bad to the bone. Semin Cell Dev Biol. 2010;21(9):951-960.

38. Goh FG, Piccinini AM, Krausgruber T, Udalova IA, Midwood KS. Transcriptional regulation of the endogenous danger signal tenascin-C: a novel autocrine loop in inflammation. J Immunol. 2010;184(5):2655-2662.

39. Nagaharu K, et al. Tenascin $\mathrm{C}$ induces epithelial-mesenchymal transition-like change accompanied by SRC activation and focal adhesion kinase phosphorylation in human breast cancer cells. Am J Pathol. 2011;178(2):754-763.

40. O'Connell JT, et al. VEGF-A and Tenascin-C produced by S100A4+ stromal cells are important for metastatic colonization. Proc Natl Acad Sci USA. 2011;108(38):16002-16007.

41. Saupe F, et al. Tenascin-C downregulates wnt inhibitor dickkopf-1, promoting tumorigenesis in a neuroendocrine tumor model. Cell Rep. 2013;5(2):482-492.

42. Xia S, Lal B, Tung B, Wang S, Goodwin CR, Laterra J. Tumor microenvironment tenascin-C promotes glioblastoma invasion and negatively regulates tumor proliferation. Neuro-oncology. 2016;18(4):507-517.

43. Jones PL, Jones FS. Tenascin-C in development and disease: gene regulation and cell function. Matrix Biol. 2000;19(7):581-596.

44. Fitzpatrick, M. Measuring cell fluorescence using ImageJ. In Fitzpatrick, M, ed. The Open Lab Book. https://theolb.readthedocs. io/en/latest/imaging/measuring-cell-fluorescence-using-imagej.html. Updated 2014. Accessed February 4, 2020

45. Kabos P, et al. Patient-derived luminal breast cancer xenografts retain hormone receptor heterogeneity and help define unique estrogen-dependent gene signatures. Breast Cancer Res Treat. 2012;135(2):415-432.

46. Schindelin J, et al. Fiji: an open-source platform for biological-image analysis. Nat Methods. 2012;9(7):676-682.

47. Johnson TD, et al. Quantification of decellularized human myocardial matrix: a comparison of six patients. Proteomics Clin Appl 2016;10(1):75-83.

48. MacLean B, et al. Skyline: an open source document editor for creating and analyzing targeted proteomics experiments. Bioin formatics. 2010;26(7):966-968.

49. Xia J, Sinelnikov IV, Han B, Wishart DS. MetaboAnalyst 3.0--making metabolomics more meaningful. Nucleic Acids Res. 2015;43(W1):W251-W257.

50. Babicki S, et al. Heatmapper: web-enabled heat mapping for all. Nucleic Acids Res. 2016;44(W1):W147-W153. 